\title{
Hamiltonian cycles in Cayley graphs whose order has few prime factors
}

\author{
K. Kutnar \\ University of Primorska, FAMNIT, Glagoljaška 8, 6000 Koper, Slovenia \\ D. Marušič \\ University of Primorska, FAMNIT, Glagoljaška 8, 6000 Koper, Slovenia \\ University of Ljubljana, PEF, Kardeljeva pl. 16, 1000 Ljubljana, Slovenia \\ D. W. Morris \\ Department of Mathematics and Computer Science, University of Lethbridge \\ Lethbridge, Alberta, TIK 3M4, Canada

\section{J. Morris} \\ Department of Mathematics and Computer Science, University of Lethbridge \\ Lethbridge, Alberta, T1K 3M4, Canada

$$
\text { P. Šparl }
$$ \\ University of Ljubljana, PEF, Kardeljeva pl. 16, 1000 Ljubljana, Slovenia
}

Received 3 October 2010, accepted 4 April 2011, published online 2 November 2011

\begin{abstract}
We prove that if Cay $(G ; S)$ is a connected Cayley graph with $n$ vertices, and the prime factorization of $n$ is very small, then $\operatorname{Cay}(G ; S)$ has a hamiltonian cycle. More precisely, if $p, q$, and $r$ are distinct primes, then $n$ can be of the form $k p$ with $24 \neq k<32$, or of the form $k p q$ with $k \leq 5$, or of the form $p q r$, or of the form $k p^{2}$ with $k \leq 4$, or of the form $k p^{3}$ with $k \leq 2$.
\end{abstract}

Keywords: Cayley graphs, hamiltonian cycles.

Math. Subj. Class.: 05C25, 05C45 


\section{Introduction}

Definition 1.1. Let $S$ be a subset of a finite group $G$. The Cayley graph $\operatorname{Cay}(G ; S)$ is the graph whose vertices are the elements of $G$, with an edge joining $g$ and $g s$, for every $g \in G$ and $s \in S$.

It was conjectured in the early 1970's that every connected Cayley graph has a hamiltonian cycle, but we are still nowhere near a resolution of this problem. (See the surveys $[6,22,27]$ for discussions of the progress that has been made.) One of the purposes of this paper is to provide some evidence for the conjecture, by establishing that all Cayley graphs on groups of small order have hamiltonian cycles. Our results are summarized in the following theorem:

Theorem 1.2. Let $G$ be a finite group. Every connected Cayley graph on $G$ has a hamiltonian cycle if $|G|$ has any of the following forms (where $p$, $q$, and $r$ are distinct primes):

1. $k p$, where $1 \leq k<32$, with $k \neq 24$,

2. $k p q$, where $1 \leq k \leq 5$,

3. $p q r$,

4. $k p^{2}$, where $1 \leq k \leq 4$,

5. $k p^{3}$, where $1 \leq k \leq 2$.

Remark 1.3 ([26]). It is also known that Cayley graphs with $p^{k}$ vertices all have hamiltonian cycles.

This work began in the 1980's as an undergraduate research project by D. Jungreis and E. Friedman at the University of Minnesota, Duluth, under the supervision of J. A. Gallian, but their results [14] were never published. (This paper is a revision and extension of the students' work; we include statements and proofs of their main results.) We consider only Cayley graphs in this paper; see [16, 18] for references to analogous work on hamiltonian cycles in more general vertex-transitive graphs with a small number of vertices.

It was originally expected that the numerous available methods would easily prove that every Cayley graph on any group of order less than, say, 100 has a hamiltonian cycle. Unfortunately, a major lesson of this work is that such an expectation is wildly incorrect. Namely, although the results here were not obtained easily, they do not even include all of the orders up to 75. More precisely, as can be seen from Fig. 1, combining Theorem 1.2 with Remark 1.3 deals with all orders less than 120 , except:

- $72=2^{3} \cdot 3^{2}=8 p^{2}$ or $24 p$,

- $96=2^{5} \cdot 3=32 p$,

- $108=2^{2} \cdot 3^{3}=36 p$ or $4 p^{3}$,

- $120=2^{3} \cdot 3 \cdot 5=24 p$.

In fact, the situation is even worse than this list would seem to indicate, because the cases $k=16, k=27$, and $k=30$ of Theorem 1.2(1) are not proved here: they were treated in the separate papers $[7,9,10]$ after a preprint of this paper was released. 


\begin{tabular}{|c|c|c|c|c|c|}
\hline 1 & $21=3 p$ & $41=p$ & $61=p$ & $81=p^{k}$ & $101=p$ \\
\hline $2=p$ & $22=2 p$ & $42=6 p$ & $62=2 p$ & $82=2 p$ & $102=6 p$ \\
\hline $3=p$ & $23=p$ & $43=p$ & $63=9 p$ & $83=p$ & $103=p$ \\
\hline $4=p^{k}$ & $24=8 p$ & $44=4 p$ & $64=p^{k}$ & $84=12 p$ & $104=8 p$ \\
\hline $5=p$ & $25=p^{2}$ & $45=9 p$ & $65=5 p$ & $85=5 p$ & $105=15 p$ \\
\hline $6=2 p$ & $26=2 p$ & $46=2 p$ & $66=6 p$ & $86=2 p$ & $106=2 p$ \\
\hline $7=p$ & $27=p^{k}$ & $47=p$ & $67=p$ & $87=3 p$ & $107=p$ \\
\hline $8=p^{k}$ & $28=4 p$ & $48=16 p$ & $68=4 p$ & $88=8 p$ & $108=? ? ?$ \\
\hline $9=p^{k}$ & $29=p$ & $49=p^{k}$ & $69=3 p$ & $89=p$ & $109=p$ \\
\hline $10=2 p$ & $30=6 p$ & $50=2 p^{2}$ & $70=2 p q$ & $90=18 p$ & $110=10 p$ \\
\hline $11=p$ & $31=p$ & $51=3 p$ & $71=p$ & $91=p q$ & $111=3 p$ \\
\hline $12=4 p$ & $32=p^{k}$ & $52=4 p$ & $72=$ ??? & $92=4 p$ & $112=16 p$ \\
\hline $13=p$ & $33=3 p$ & $53=p$ & $73=p$ & $93=3 p$ & $113=p$ \\
\hline $14=2 p$ & $34=2 p$ & $54=2 p^{3}$ & $74=2 p$ & $94=2 p$ & $114=6 p$ \\
\hline $15=3 p$ & $35=p q$ & $55=p q$ & $75=3 p^{2}$ & $95=5 p$ & $115=5 p$ \\
\hline $16=p^{k}$ & $36=4 p^{2}$ & $56=8 p$ & $76=4 p$ & $96=? ? ?$ & $116=4 p$ \\
\hline $17=p$ & $37=p$ & $57=3 p$ & $77=p q$ & $97=p$ & $117=9 p$ \\
\hline $18=2 p^{2}$ & $38=2 p$ & $58=2 p$ & $78=6 p$ & $98=2 p^{2}$ & $118=2 p$ \\
\hline $19=p$ & $39=3 p$ & $59=p$ & $79=p$ & $99=9 p$ & $119=7 p$ \\
\hline $20=4 p$ & $40=8 p$ & $60=12 p$ & $80=16 p$ & $100=4 p^{2}$ & $120=? ? ? ?$ \\
\hline
\end{tabular}

Figure 1: Factorizations of orders up to 120.

\section{Outline of the paper}

Most of the cases of Theorem 1.2 are known (including all of the cases where $k=1$ ). For example, C.C. Chen and N. Quimpo [5] proved that Cayley graphs of order $p q$ are hamiltonian (in fact, edge-hamiltonian), and D. Li [19] proved that Cayley graphs of order $p q r$ are hamiltonian. (However, the latter result is in Chinese, so we provide a proof.) The following list of the paper's sections enumerates the main cases that need to be considered.
$\S 2$ Preliminaries
$\S 5$ Groups of order $4 p^{2}$
$\S 8$ Groups of order $2 p^{3}$
$\S 3$ Groups of order $8 p$
$\S 6$ Groups of order $p q r$
$\S 9$ Groups of order $18 p$
$\S 4$ Groups of order $3 p^{2}$
$\S 7$ Groups of order $4 p q$

\section{Preliminaries}

\section{A Outline of the proof of Theorem 1.2}

Here is a description of how the results of this paper combine to prove Theorem 1.2. 
(1) If $k \in\{2,3,5,6,7,10,11,13,14,17,19,22,23,26,29,31\}$, then $k$ is either prime or twice a prime, so $k p$ is of the form $p q, 2 p q, p^{2}$, or $2 p^{2}$. These cases are treated below, in (2a), (2b), (4a), and (4b), respectively, so we need only consider the other values of $k$. Also, we note that the proofs of (2)-(5) make no use of (1), other than the cases $4 p$ and $8 p$, so we are free to employ any and all other parts of the theorem in establishing the cases of (1) (other than $4 p$ and $8 p$ ).

$1 p$ : Groups of prime order are abelian, so Lemma 2.1 applies.

$4 p$ : See Corollary 2.17.

$8 p$ : See Proposition 3.2.

$9 p$ : Corollary 2.3 applies unless $p=2$. If $p=2$, then $|G|$ is of the form $2 p^{2}$.

$12 p$ : $|G|$ is of the form $8 p$ (if $p=2$ ) or $4 p^{2}$ (if $p=3$ ) or $4 p q$ (if $p>3$ ).

$15 p$ : $|G|$ is of the form $3 p^{2}$ (if $p=5$ ) or $3 p q$ (otherwise).

16p: See [7].

18p: See Proposition 9.1.

$20 p:|G|$ is of the form $4 p^{2}$ (if $p=5$ ) or $4 p q$ (otherwise).

$21 p:|G|$ is of the form $3 p^{2}$ (if $p=7$ ) or $3 p q$ (otherwise).

$25 p$ : Corollary 2.3 applies unless $p \in\{2,3\}$. In the exceptional cases, $|G|$ is of the form $k p^{2}$ with $1 \leq k \leq 4$.

$27 p$ : See [9].

$28 p$ : $|G|$ is of the form $4 p^{2}$ (if $p=7$ ) or $4 p q$ (otherwise).

$30 p$ : See [10].

(2) Assume $|G|=k p q$ with $1 \leq k \leq 5$.

(a) If $k=1$, then $[G, G]$ is cyclic of prime order, so Theorem 2.2 applies.

(b) If $k=2$, see Proposition 6.1.

(c) If $k=3$, see Corollary 6.3 .

(d) If $k=4$, see Proposition 7.2.

(e) If $k=5$, see Corollary 6.4 .

(3) Assume $|G|=p q r$. See Proposition 6.2 (or [19]).

(4) Assume $|G|=k p^{2}$ with $1 \leq k \leq 4$.

(a) If $k=1$, then $|G|=p^{2}$, so $G$ is abelian. Hence, Lemma 2.1 applies.

(b) If $k=2$, see Corollary 2.24.

(c) If $k=3$, see Proposition 4.1 .

(d) If $k=4$, see Proposition 5.3.

(5) Assume $|G|=k p^{3}$ with $1 \leq k \leq 2$.

(a) If $k=1$, then $|G|=p^{3}$ is a prime power, so Remark 1.3 applies.

(b) If $k=2$, see Proposition 8.1 . 


\section{B Some basic results on Cayley graphs of small order}

It is very easy to see that Cayley graphs on abelian groups are hamiltonian (in fact, they are edge-hamiltonian [5] and are usually hamiltonian connected [4]):

Lemma 2.1 ([4]). If $G$ is abelian, then every connected Cayley graph on $G$ has a hamiltonian cycle.

The following generalization handles many groups of small order:

Theorem 2.2 (Keating-Witte [15]). If the commutator subgroup $[G, G]$ of $G$ is a cyclic pgroup, then every connected Cayley graph on $G$ has a hamiltonian cycle.

For ease of reference, we record a well-known (and easy) consequence of this theorem.

Corollary 2.3. If $|G|=p^{2} q$, where $p$ and $q$ are primes with $p^{2} \not \equiv 1(\bmod q)$, then every connected Cayley graph on $G$ has a hamiltonian cycle.

Proof. We may assume $p \neq q$, for otherwise $|G|=p^{3}$ is a prime power, so Remark 1.3 applies.

Let $Q$ be a Sylow $q$-subgroup of $G$. From Sylow's Theorem (2.33), we know that $Q$ is normal in $G$. The quotient group $G / Q$, being of order $p^{2}$, must be abelian. Therefore $[G, G] \subset Q$ is cyclic of order $q$ or 1 , so Theorem 2.2 applies.

The proof of Remark 1.3 actually yields the following stronger result:

Corollary 2.4 ([21, Cor. 3.3]). Suppose

- $S$ is a generating set of $G$,

- $N$ is a normal p-subgroup of $G$, and

- $s t^{-1} \in N$, for all $s, t \in S$.

Then $\operatorname{Cay}(G ; S)$ has a hamiltonian cycle.

\section{C Factor Group Lemma}

When proving the various parts of Theorem 1.2, we will implicitly assume, by induction on $|G|$, that if $N$ is any nontrivial, normal subgroup of $G$, then every connected Cayley graph on $G / N$ has a hamiltonian cycle. (Similarly, we also assume that if $H$ is any proper subgroup of $G$, then every connected Cayley graph on $H$ has a hamiltonian cycle.) Thus it is very useful to know when we can lift hamiltonian cycles from a quotient graph to the original Cayley graph. Here are a few well-known results of this type.

Notation 2.5. For $s_{1}, s_{2}, \ldots, s_{n} \in S \cup S^{-1}$, we use

$$
\left(s_{1}, s_{2}, s_{3}, \ldots, s_{n}\right)
$$

to denote the walk in $\operatorname{Cay}(G ; S)$ that visits (in order) the vertices

$$
e, s_{1}, s_{1} s_{2}, s_{1} s_{2} s_{3}, \ldots, s_{1} s_{2} \cdots s_{n} .
$$

Also,

- $\left(s_{1}, s_{2}, s_{3}, \ldots, s_{n}\right)^{k}$ denotes the walk that is obtained from the concatenation of $k$ copies of $\left(s_{1}, s_{2}, s_{3}, \ldots, s_{n}\right)$, and 
- $\left(s_{1}, s_{2}, s_{3}, \ldots, s_{n}\right) \#$ denotes the walk $\left(s_{1}, s_{2}, s_{3}, \ldots, s_{n-1}\right)$ obtained by deleting the last term of the sequence.

The following observation is elementary.

\section{Lemma 2.6. Suppose}

- $S$ is a generating set of $G$,

- $H$ is a cyclic subgroup of $G$, with index $|G: H|=n$,

- $s_{1}, s_{2}, \ldots, s_{n}$ is a sequence of $n$ elements of $S \cup S^{-1}$, such that

$\circ$ the elements $e, s_{1}, s_{1} s_{2}, s_{1} s_{2} s_{3}, \ldots, s_{1} s_{2} \cdots s_{n-1}$ are all in different right cosets of $H$, and

$\circ$ the product $s_{1} s_{2} s_{3} \cdots s_{n}$ is a generator of $H$.

Then $\left(s_{1}, \ldots, s_{n}\right)^{|H|}$ is a hamiltonian cycle in $\operatorname{Cay}(G ; S)$.

The assumptions on the sequence $s_{1}, s_{2}, \ldots, s_{n}$ can also be expressed by saying that a certain quotient multigraph has a hamiltonian cycle:

Definition 2.7. If $H$ is any subgroup of $G$, then $H \backslash \operatorname{Cay}(G ; S)$ denotes the multigraph in which:

- the vertices are the right cosets of $H$, and

- there is an edge joining $H g_{1}$ and $H g_{2}$ for each $s \in S \cup S^{-1}$, such that $g_{1} s \in H g_{2}$.

Thus, if there are two different elements $s_{1}$ and $s_{2}$ of $S \cup S^{-1}$, such that $g_{1} s_{1}$ and $g_{1} s_{2}$ are both in $\mathrm{Hg}_{2}$, then the vertices $\mathrm{Hg}_{1}$ and $\mathrm{Hg}_{2}$ are joined by a double edge.

When the cyclic subgroup $H$ is normal, we have the following well-known special case:

Corollary 2.8 ("Factor Group Lemma"). Suppose

- $S$ is a generating set of $G$,

- $N$ is a cyclic, normal subgroup of $G$,

- $\left(s_{1} N, \ldots, s_{n} N\right)$ is a hamiltonian cycle in $\operatorname{Cay}(G / N ; S)$, and

- the product $s_{1} s_{2} \cdots s_{n}$ generates $N$.

Then $\left(s_{1}, \ldots, s_{n}\right)^{|N|}$ is a hamiltonian cycle in $\operatorname{Cay}(G ; S)$.

When $|H|$ (or $|N|$ ) is prime, it is generated by any of its nontrivial elements. So, in order to know that there is a hamiltonian cycle for which the product $s_{1} s_{2} \cdots s_{n}$ generates $H$, it suffices to know that there are two hamiltonian cycles that differ in only one edge:

Corollary 2.9. Suppose

- $S$ is a generating set of $G$,

- $H$ is a subgroup of $G$, such that $|H|$ is prime,

- the quotient multigraph $H \backslash \operatorname{Cay}(G ; S)$ has a hamiltonian cycle $C$, and

- $C$ uses some double edge of $H \backslash \mathrm{Cay}(G ; S)$. 
Then there is a hamiltonian cycle in $\operatorname{Cay}(G ; S)$.

Definition 2.10. We say that a generating set $S$ of a group $G$ is minimal if no proper subset of $S$ generates $G$.

\section{Corollary 2.11. Suppose}

- $N$ is a normal subgroup of $G$, such that $|N|$ is prime,

- the image of $S$ in $G / N$ is a minimal generating set of $G / N$,

- there is a hamiltonian cycle in $\operatorname{Cay}(G / N ; S)$, and

- $s \equiv t(\bmod N)$ for some $s, t \in S \cup S^{-1}$ with $s \neq t$.

Then there is a hamiltonian cycle in $\operatorname{Cay}(G ; S)$.

We will also use the following generalization of Lemma 2.6:

Lemma 2.12 ([26, Lem. 5.1]). Suppose

- $K$ is a normal subgroup of a subgroup $H$ of $G$,

- $\left(s_{1}, s_{2}, \ldots, s_{n}\right)$ is a hamiltonian cycle in the quotient $H \backslash \operatorname{Cay}(G ; S)$, and

- the product $s_{1} s_{2} \cdots s_{n}$ generates $H / K$.

Then $\left(s_{1}, s_{2}, \ldots, s_{n}\right)^{|H / K|}$ is a hamiltonian cycle in $K \backslash \operatorname{Cay}(G ; S)$.

The theory of "voltage graphs" [12, Thm. 2.1.3, p. 63] (or see [2, Thm. 5.2]) provides a method for applying Lemma 2.6. Here is one example that we will use:

Theorem 2.13 (Locke-Witte, c.f. [20, Prop. 3.3]). Suppose

- $\operatorname{Cay}(G ; S)$ is connected,

- $N$ is a normal subgroup of $G$,

- $|N|$ is prime, and

- for some $k$, $\operatorname{Cay}(G / N ; S)$ is isomorphic to either

- $\operatorname{Cay}\left(\mathbb{Z}_{4 k} ;\{1,2 k\}\right)$ (a non-bipartite Möbius ladder), or

○ $\operatorname{Cay}\left(\mathbb{Z}_{2 k} \times \mathbb{Z}_{2} ;\{(1,0),(0,1)\}\right)$ (a bipartite prism), with $2 k \neq \equiv 1(\bmod |N|)$.

Then some hamiltonian cycle in $\operatorname{Cay}(G / N ; S)$ lifts to a hamiltonian cycle in $\operatorname{Cay}(G ; S)$.

\section{D Applications of Lemma 2.6}

For future reference, we record some special cases of Lemma 2.6. Although the hypotheses of these results are very restrictive (and rather complicated), they will be used many times.

Lemma 2.14 (Jungreis-Friedman [14, Lem. 6.1]). Let $\left\{s_{1}, s_{2}\right\}$ generate the group $G$. If

- $2\left|s_{1}\right| \cdot\left|\left[s_{1}, s_{2}\right]\right|=|G|$,

- $s_{2} \notin\left\langle s_{1}\right\rangle\left\langle\left[s_{1}, s_{2}\right]\right\rangle$,

- $\left\langle\left[s_{1}, s_{2}\right]\right\rangle \cap\left\langle s_{1}\right\rangle=\{e\}$, and

- $\left\langle\left[s_{1}, s_{2}\right]\right\rangle \cap\left(s_{2}^{-1}\left\langle s_{1}\right\rangle s_{2}\right)=\{e\}$, 
then $\operatorname{Cay}\left(G ;\left\{s_{1}, s_{2}\right\}\right)$ has a hamiltonian cycle.

Proof. For convenience, let $\gamma=\left[s_{1}, s_{2}\right]=s_{1}^{-1} s_{2}^{-1} s_{1} s_{2}$. We claim that

$$
\left(s_{1}^{\left|s_{1}\right|-1}, s_{2}^{-1}, s_{1}^{-\left(\left|s_{1}\right|-1\right)}, s_{2}\right)^{|\gamma|}
$$

is a hamiltonian cycle. This will follow from Lemma 2.6 if we show that the vertices of the walk $\left(s_{1}^{\left|s_{1}\right|-1}, s_{2}^{-1}, s_{1}^{-\left(\left|s_{1}\right|-1\right)}\right)$ are all in different right cosets of $\langle\gamma\rangle$.

Note that the vertices in this walk are all in $\left\langle s_{1}\right\rangle$ or $s_{1}^{-1} s_{2}^{-1}\left\langle s_{1}\right\rangle$, and that

$$
\langle\gamma\rangle s_{1}^{-1} s_{2}^{-1}\left\langle s_{1}\right\rangle=\langle\gamma\rangle\left(s_{1}^{-1} s_{2}^{-1} s_{1} s_{2}\right) s_{2}^{-1} s_{1}^{-1}\left\langle s_{1}\right\rangle=\langle\gamma\rangle s_{2}^{-1}\left\langle s_{1}\right\rangle
$$

- Since $\langle\gamma\rangle \cap\left\langle s_{1}\right\rangle=\{e\}$, we know that all of the elements of $\left\langle s_{1}\right\rangle$ are in different right cosets.

- Since $\langle\gamma\rangle \cap s_{2}^{-1}\left\langle s_{1}\right\rangle s_{2}=\{e\}$, we know that all of the elements of $s_{2}^{-1}\left\langle s_{1}\right\rangle$ are in different right cosets. So (2.15) implies that all of the elements of $s_{1}^{-1} s_{2}^{-1}\left\langle s_{1}\right\rangle$ are in different right cosets.

- Since $s_{2}^{-1} \notin\langle\gamma\rangle\left\langle s_{1}\right\rangle$, we know that

$$
\langle\gamma\rangle\left\langle s_{1}\right\rangle \cap\langle\gamma\rangle s_{2}^{-1}\left\langle s_{1}\right\rangle=\emptyset
$$

So (2.15) implies that none of the elements of $\left\langle s_{1}\right\rangle$ are in the same right coset as any element of $s_{1}^{-1} s_{2}^{-1}\left\langle s_{1}\right\rangle$.

Lemma 2.14 will be used many times; here is an example.

Corollary 2.16. If $G \cong \mathbb{Z}_{3^{k}} \ltimes\left(\mathbb{Z}_{2} \times \mathbb{Z}_{2}\right)$, for some $k \in \mathbb{Z}^{+}$, then every connected Cayley graph on $G$ has a hamiltonian cycle.

Proof. We may assume $\mathbb{Z}_{3^{k}}$ acts nontrivially on $\mathbb{Z}_{2} \times \mathbb{Z}_{2}$, for otherwise $G$ is abelian, so Theorem 2.2 applies. Then $\# S=2$, and some element $s_{1}$ of $S$ generates (a conjugate of) $\mathbb{Z}_{3^{k}}$. The other element $s_{2}$ of $S$ is of the form $s_{1}^{i} y$ with $y \in \mathbb{Z}_{2} \times \mathbb{Z}_{2}$, so we have

$$
\left[s_{1}, s_{2}\right]=\left[s_{1}, y\right] \in\left(\mathbb{Z}_{2} \times \mathbb{Z}_{2}\right)-\langle y\rangle,
$$

so it is easy to verify the hypotheses of Lemma 2.14.

Corollary 2.17. If $|G|=4 p$, where $p$ is prime, then every connected Cayley graph on $G$ has a hamiltonian cycle.

Proof. Corollary 2.3 applies unless $p=3$. However, if $p=3$, then either the Sylow 3subgroup is normal, so the argument of Corollary 2.3 applies, or $G \cong A_{4} \cong \mathbb{Z}_{3} \ltimes\left(\mathbb{Z}_{2} \times \mathbb{Z}_{2}\right)$, so Corollary 2.16 applies.

Lemma 2.18 (Jungreis-Friedman [14, Lem. 7.1]). Let $S$ be a minimal generating set for the group $G$. If there exist two distinct generators $s_{1}, s_{2} \in S$ such that:

- $\left|s_{1} s_{2}\right|=|G| /\left|\left\langle S-\left\{s_{1}\right\}\right\rangle\right|$,

- $\left\langle s_{1} s_{2}\right\rangle \cap\left\langle S-\left\{s_{1}\right\}\right\rangle=\{e\}$, and

- there is a hamiltonian cycle in Cay $\left(\left\langle S-\left\{s_{1}\right\}\right\rangle ; S-\left\{s_{1}\right\}\right)$, 
then there is a hamiltonian cycle in $\operatorname{Cay}(G ; S)$.

Proof. Let $\left(t_{i}\right)_{i=1}^{n}$ be a hamiltonian cycle in $\operatorname{Cay}\left(\left\langle S-\left\{s_{1}\right\}\right\rangle ; S-\left\{s_{1}\right\}\right)$. Since $S$ is a minimal generating set for $G$, we know that $s_{2}$ or its inverse must appear somewhere in this cycle, and by choosing a different starting point if necessary, and reversing the cycle if necessary, we can assume without loss of generality that $t_{n}=s_{2}^{-1}$. Then $t_{1} t_{2} \ldots t_{n-1}=$ $s_{2}$.

Since $\left\langle s_{1} s_{2}\right\rangle \cap\left\langle S-\left\{s_{1}\right\}\right\rangle=\{e\}$, conjugating by $s_{2}^{-1}$ tells us that $\left\langle s_{2} s_{1}\right\rangle \cap\left\langle S-\left\{s_{1}\right\}\right\rangle$ is also trivial. So the elements of $\left\langle S-\left\{s_{1}\right\}\right\rangle$ are all in different right cosets of $\left\langle s_{2} s_{1}\right\rangle$. Therefore Lemma 2.6 tells us that

$$
\left(\left(t_{i}\right)_{i=1}^{n-1}, s_{1}\right)^{\left|s_{1} s_{2}\right|}
$$

is a hamiltonian cycle in $\operatorname{Cay}(G ; S)$.

Corollary 2.19. Let $S$ be a minimal generating set for the group $G$. If there exist two distinct generators $s_{1}, s_{2} \in S$, such that

- $\operatorname{Cay}\left(\left\langle S-\left\{s_{1}\right\}\right\rangle ; S-\left\{s_{1}\right\}\right)$ has a hamiltonian cycle, and

- $\left|s_{1} s_{2}\right|=|G| /\left|\left\langle S-\left\{s_{1}\right\}\right\rangle\right|$ is prime,

then there is a hamiltonian cycle in $\operatorname{Cay}(G ; S)$.

Proof. In order to apply Lemma 2.18, we need only show that $\left\langle s_{1} s_{2}\right\rangle \cap\left\langle S-\left\{s_{1}\right\}\right\rangle=\{e\}$. Suppose, to the contrary, that $\left\langle S-\left\{s_{1}\right\}\right\rangle$ contains a nontrivial element of $\left\langle s_{1} s_{2}\right\rangle$. Since $\left|s_{1} s_{2}\right|$ is prime, this implies that $s_{1} s_{2} \in\left\langle S-\left\{s_{1}\right\}\right\rangle$. But, since $s_{1}$ and $s_{2}$ are distinct, we also have $s_{2} \in\left\langle S-\left\{s_{1}\right\}\right\rangle$. Therefore $s_{1} \in\left\langle S-\left\{s_{1}\right\}\right\rangle$, contradicting the minimality of $S$.

\section{E Groups of dihedral type}

Notation 2.20. We use $D_{2 n}$ and $Q_{4 n}$ to denote the dihedral group of order $2 n$ and the generalized quaternion group of order $4 n$, respectively. That is,

$$
D_{2 n}=\left\langle f, x \mid f^{2}=x^{n}=e, f x f=x^{-1}\right\rangle
$$

and

$$
Q_{4 n}=\left\langle f, x \mid x^{2 n}=e, f^{2}=x^{n}, f^{-1} x f=x^{-1}\right\rangle .
$$

\section{Definition 2.21.}

- A group $G$ is of dihedral type if it has

$\circ$ an abelian subgroup $A$ of index 2 , and

$\circ$ an element $f$ of order 2 (with $f \notin A$ ),

such that $f$ inverts every element of $A$ (i.e., $f^{-1} a f=a^{-1}$ for all $a \in A$ ).

- A group $G$ is of quaternion type if it has

$\circ$ an abelian subgroup $A$ of index 2 , and

$\circ$ an element $f$ of order 4 , 
such that $f$ inverts every element of $A$.

Thus, dihedral groups are the groups of dihedral type in which $A$ is cyclic, while generalized quaternion groups are the groups of quaternion type in which $A$ is cyclic.

It is not very difficult to show that Cayley graphs on dihedral groups of small order are hamiltonian:

Lemma 2.22 (Witte [25, Prop. 5.5]). If n has at most three distinct prime factors, then every connected Cayley graph on $D_{2 n}$ has a hamiltonian cycle.

A similar argument also yields a result for other groups of dihedral type:

Proposition 2.23 (Jungreis-Friedman [14, Thm. 5.4]). If $G=\mathbb{Z}_{2} \ltimes A$ is of dihedral type, and $|A|$ is the product of at most three primes (not necessarily distinct), then every connected Cayley graph on $G$ has a hamiltonian cycle.

Proof. Let $S$ be a minimal generating set of $G$. Since every element of $f A$ inverts $A$, it is easy to see that we may assume $S \cap A=\emptyset$ (cf. [25, Thm. 5.3]), and that $f \in S$.

- If $A$ is a $p$-group, then Corollary 2.4 applies.

- If $A$ is cyclic, then $G$ is dihedral, so Lemma 2.22 applies.

Thus, we may assume $A=\mathbb{Z}_{p} \times \mathbb{Z}_{p} \times \mathbb{Z}_{q}$, where $p$ and $q$ are distinct primes.

Note that $f S-\{e\}$ must be a minimal generating set of $A$.

Case 1. Assume $f S$ contains an element $x$ of order $p$. Then $f S-\{x\}$ must generate a subgroup of order $p q$ (necessarily cyclic), so $\langle S-\{f x\}\rangle \cong D_{2 p q}$; let $\left(s_{1}, s_{2}, \ldots, s_{2 p q}\right)$ be a Hamiltonian cycle in Cay $\left(D_{2 p q} ; S-\{f x\}\right)$. We may assume $s_{2 p q}=f$. The vertices of the path $\left(s_{1}, s_{2}, \ldots, s_{2 p q}\right) \#$ are all in different right cosets of $\langle x\rangle$, so Lemma 2.6 implies that $\left(\left(s_{1}, s_{2}, \ldots, s_{2 p q}\right) \#, f x\right)^{p}$ is a hamiltonian cycle in $\operatorname{Cay}(G ; S)$.

Case 2. Assume $f S$ does not contain any element of order $p$. Then $S=\left\{f, f_{1}, f_{2}\right\}$, where $f f_{1}$ and $f f_{2}$ both have order $p q$ (and $\left\langle f f_{1}, f f_{2}\right\rangle=A=\mathbb{Z}_{p} \times \mathbb{Z}_{p} \times \mathbb{Z}_{q}$ ). We may assume $p \geq 3$, for otherwise $[G, G]=\mathbb{Z}_{q}$, so Theorem 2.2 applies. Then, since at least one of any four consecutive integers is relatively prime to $p q$, there exists $k \in\{0,1,2, \ldots, p\}$, such that $\left(f_{2} f\right)^{p}\left(f f_{1}\right)^{k}$ generates $\left\langle f f_{1}\right\rangle$. This means that $\left(f_{2} f\right)^{p-k}\left(f_{2} f_{1}\right)^{k}$ generates $\left\langle f f_{1}\right\rangle$, so Corollary 2.8 implies that $\left(\left(f_{2} f\right)^{p-k},\left(f_{2} f_{1}\right)^{k}\right)$ is a hamiltonian cycle in Cay $\left(A ;\left\{f_{2} f\right.\right.$, $\left.\left.f_{2} f_{1}\right\}\right)$. Then it is clear that $\left(\left(f_{2}, f\right)^{p-k},\left(f_{2}, f_{1}\right)^{k}\right)$ is a hamiltonian cycle in Cay $(G ;\{f$, $\left.\left.f_{1}, f_{2}\right\}\right)$.

Corollary 2.24. If $|G|=2 p^{2}$, where $p$ is prime, then every connected Cayley graph on $G$ has a hamiltonian cycle.

Proof. Either $[G, G]$ is cyclic of order $p$ (so Theorem 2.2 applies) or $G$ is of dihedral type, so Proposition 2.23 applies.

Corollary 2.25 (Jungreis-Friedman cf. [14, Thm. 5.1]). If $n$ is the product of at most three primes (not necessarily distinct), then every connected Cayley graph on any group of quaternion type of order $4 n$ has a hamiltonian cycle. 
Proof. Let Cay $(G ; S)$ be such a Cayley graph, and assume, without loss of generality, that $S$ is a minimal generating set for $G$. Let $A$ be an abelian subgroup of index 2 in $G$, and let $f \in S$, with $f \notin A$. Then $\left\langle f^{2}\right\rangle$ is a normal subgroup of order 2 in $G$. Furthermore, $G /\left\langle f^{2}\right\rangle$ is of dihedral type, so Proposition 2.23 implies there is a hamiltonian cycle in Cay $\left(G /\left\langle f^{2}\right\rangle ; S\right)$. Therefore, Corollary 2.11 applies with $s=f=t^{-1}$.

Remark 2.26. If $G$ is a group of dihedral type, and $|G|$ is divisible by 4 , then B. Alspach, C. C. Chen, and M. Dean [3] have shown that every connected Cayley graph on $G$ has a hamiltonian cycle. In fact, the Cayley graphs are hamiltonian connected (or hamiltonian laceable when they are bipartite).

\section{F Generator in a cyclic, normal subgroup}

The following observation is well known.

Lemma 2.27. Let $S$ generate $G$ and let $s \in S$, such that $\langle s\rangle \triangleleft G$. If

- $\operatorname{Cay}(G /\langle s\rangle ; S)$ has a hamiltonian cycle, and

- either

1. $s \in Z(G)$, or

2. $Z(G) \cap\langle s\rangle=\{e\}$, or

3. $|s|$ is prime,

then $\operatorname{Cay}(G ; S)$ has a hamiltonian cycle.

Proof. Let $\left(s_{1}, s_{2}, \ldots, s_{n}\right)$ be a hamiltonian cycle in Cay $(G /\langle s\rangle ; S)$, and let $k=\mid s_{1} s_{2} \ldots$ $s_{n} \mid$, so $\left(s_{1}, s_{2}, \ldots, s_{n}\right)^{k}$ is a cycle in $\operatorname{Cay}(G ; S)$.

(1) Since $s \in Z(G)$, it is easy to see that $\operatorname{Cay}(G ; S)$ contains a spanning subgraph isomorphic to the Cartesian product $P_{n} \times C_{|s|}$ of a path with $n$ vertices and a cycle with $|s|$ vertices. Since it is easy to see that this Cartesian product is hamiltonian [4, Cor. on p. 29], we conclude that $\operatorname{Cay}(G ; S)$ has a hamiltonian cycle.

(2) Let $m=|G| /(n k)$. We claim that

$$
\left(s^{m-1}, s_{1}, s^{m-1}, s_{2}, s^{m-1}, \ldots, s^{m-1}, s_{n}\right)^{k}
$$

is a hamiltonian cycle in $\operatorname{Cay}(G ; S)$.

Let

$$
g_{i}=\left(s_{1} s_{2} \cdots s_{i}\right)^{-1} \text { for } 0 \leq i \leq n, \text { so } g_{i} g_{i+1}^{-1}=s_{i+1},
$$

and note that, since $\left(s_{1}, s_{2}, \ldots, s_{n}\right)$ is a hamiltonian cycle, we know that

$$
\left\{1, g_{1}, g_{2}, \ldots, g_{n-1}\right\} \text { is a complete set of coset representatives for }\langle s\rangle \text { in } G \text {. }
$$

Then, for any $h \in G$,

$$
\left\{h, g_{1} h, g_{2} h, \ldots, g_{n-1} h\right\} \text { is also a set of coset representatives. }
$$

Also, since $\langle s\rangle$ is abelian, we know that if $x$ and $y$ are elements in the same coset of $\langle s\rangle$, then $s^{x}=s^{y}$. Thus, for any $t \in\langle s\rangle$, we have

$$
\left\{t, t^{g_{1}}, t^{g_{2}}, \ldots, t^{g_{n-1}}\right\}=\left\{t^{h}, t^{g_{1} h}, t^{g_{2} h}, \ldots, t^{g_{n-1} h}\right\},
$$


so

$$
t t^{g_{1}} t^{g_{2}} \cdots t^{g_{n-1}}=t^{h} t^{g_{1} h} t^{g_{2} h} \cdots t^{g_{n-1} h},
$$

because both products have exactly the same factors (but possibly in a different order). Since the right-hand product is $\left(t t^{g_{1}} t^{g_{2}} \cdots t^{g_{n-1}}\right)^{h}$, and $h$ is an arbitrary element of $G$, we conclude that $t t^{g_{1}} t^{g_{2}} \cdots t^{g_{n-1}} \in Z(G)$. Since $Z(G)$ has trivial intersection with $\langle s\rangle$, this implies that

$$
t t^{g_{1}} t^{g_{2}} \cdots t^{g_{n-1}}=e .
$$

Therefore

$$
\begin{aligned}
& \quad\left(s^{m-1}\right) s_{1}\left(s^{m-1}\right) s_{2} \cdots\left(s^{m-1}\right) s_{n}= \\
& \quad=\left(\left(s^{m-1}\right)\left(s^{m-1}\right)^{g_{1}}\left(s^{m-1}\right)^{g_{2}} \cdots\left(s^{m-1}\right)^{g_{n-1}}\right) g_{n}^{-1}=g_{n}^{-1} .
\end{aligned}
$$

Therefore

$$
\left(\left(s^{m-1}\right) s_{1}\left(s^{m-1}\right) s_{2} \cdots\left(s^{m-1}\right) s_{n}\right)^{k}=g_{n}^{-k}=\left(s_{1} s_{2} \cdots s_{n}\right)^{k}=e,
$$

so the walk is closed. Furthermore, since $m=\left|\langle s\rangle /\left\langle g_{n}\right\rangle\right|$, it is clear that the walk visits every element of $\langle s\rangle$, and it is similarly easy to see that it visits every element of all of the other cosets. So it visits every element of $G$.

Since it is also a closed walk of the correct length, we conclude that it is a hamiltonian cycle.

(3) Since $|s|$ is prime, either (1) or (2) must apply.

The following related result is much less obvious.

Theorem 2.28 (Alspach [2, Thm. 3.7]). Suppose

- $S$ is a generating set of $G$,

- $s \in S$,

- $\langle s\rangle \triangleleft G$,

- $|G:\langle s\rangle|$ is odd, and

- Cay $(G /\langle s\rangle ; S)$ has a hamiltonian cycle.

Then $\operatorname{Cay}(G ; S)$ has a hamiltonian cycle.

A well-known theorem of B. Alspach [1] describes exactly which generalized Petersen graphs have a hamiltonian cycle. We need only the following consequence of this very precise result.

Theorem 2.29 (B. Alspach [1]). Suppose $X$ is a generalized Petersen graph that is connected, and has $2 n$ vertices. If $n \not \equiv 0(\bmod 4)$ and $n \not \equiv 5(\bmod 6)$, then $X$ has a hamiltonian cycle.

\section{G A few small groups}

For future reference, we record the existence of hamiltonian cycles in every connected Cayley graph on the groups $S_{4}, A_{4} \times \mathbb{Z}_{2}, A_{4} \times \mathbb{Z}_{3}$, and $A_{5}$. Only a few non-isomorphic Cayley graphs arise on each group, and a computer search could quickly find a hamiltonian cycle in each of them, so, for brevity, we omit some details of the proofs. 
Lemma 2.30 ([14, Thm. 8.2]). Every connected Cayley graph on the symmetric group $S_{4}$ has a hamiltonian cycle.

Proof. Suppose $S$ is a minimal generating set of $S_{4}$. Note that $S$ must contain an odd permutation; that is, $S$ contains either a 2-cycle or 4-cycle.

Case 1. Assume $\# S=2$. Write $S=\{a, b\}$.

If $a$ is a 4-cycle, then we may assume

$$
a=(1,2,3,4) \text { and } b \in\{(1,2),(1,2,3),(1,2,4,3)\} .
$$

In each case, Lemma 2.14 provides a hamiltonian cycle in $\operatorname{Cay}\left(S_{4} ; S\right)$.

Now suppose $S$ contains no 4 -cycles. Then we may assume $a=(1,2)$ and $b=$ $(2,3,4)$. In this case, a hamiltonian cycle is given by $\left(\left(a, b^{2}\right)^{2},\left(a, b^{-2}\right)^{2}\right)^{2}$.

Case 2. Assume $\# S \geq 3$. Since $S$ is minimal, it is easy to see that $\# S=3$; write $S=\{a, b, c\}$.

Subcase 2.1. Assume $a=(1,2)(3,4)$. Let $N$ be the normal subgroup of order 4 that contains $a$. Then, since $\langle S\rangle=S_{4}$, we have $\langle b, c\rangle N=S_{4}$. Furthermore, since the action of $S_{4} / N$ on $N$ is irreducible, the minimality of $S$ implies $\langle b, c\rangle \cap N$ is trivial. So $\langle b, c\rangle \cong S_{4} / N \cong S_{3}$. Then, conjugating by a power of $(1,3,2,4)$ (which centralizes $a$ ), there is no harm in assuming that $\langle b, c\rangle=S_{3}$. So we may assume $\{b, c\}$ is either

$$
\{(1,2),(2,3)\} \text { or }\{(1,3),(2,3)\} \text { or }\{(1,2,3),(1,2)\} \text { or }\{(1,2,3),(2,3)\} \text {. }
$$

If $(2,3) \in S$, then Lemma 2.18 applies with $s_{1}=(1,2)(3,4)$ and $s_{2}=(2,3)$. In the remaining case, let $b=(1,2,3)$ and $c=(1,2)$, and let $L$ be any hamiltonian path in $\operatorname{Cay}\left(A_{4} ;\{a, b\}\right)$ from $e$ to $b$. Then $(L, c)^{2}$ is a hamiltonian cycle in $\operatorname{Cay}\left(S_{4} ; S\right)$.

Subcase 2.2. Assume $S$ does not contain any even permutation of order 2 . Then, since $S$ is a 3-element, minimal generating set, it is not difficult to see that $S$ cannot contain a 4 -cycle. So $S$ consists entirely of 2 -cycles and 3-cycles. However, it is known that there is a hamiltonian cycle in $\operatorname{Cay}\left(S_{n}: S\right)$ whenever $S$ consists entirely of 2-cycles (see the discussion and references on p. 622 of [23]), so we may assume that $S$ contains at least one 3 -cycle. Then, up to automorphism, we have

$$
S=\{(1,2,3),(1,2,4),(1,2)\} .
$$

Let $a=(1,2,3), b=(1,2,4)$, and $c=(1,2)$, and let $L$ be any hamiltonian path in $\operatorname{Cay}\left(A_{4} ;\{a, b\}\right)$ from $e$ to $b$. Then $(L, c)^{2}$ is a hamiltonian cycle in $\operatorname{Cay}\left(S_{4} ; S\right)$.

We actually need only the cases $p=2$ and $p=3$ of the following result, but the general case is no more difficult to prove.

Lemma 2.31 (Jungreis-Friedman [14, Thm. 7.4]). If p is prime, then every connected Cayley graph on $A_{4} \times \mathbb{Z}_{p}$ has a hamiltonian cycle.

Proof. Suppose $S$ is a minimal generating set for the group $A_{4} \times \mathbb{Z}_{p}$, and let $z$ be a generator of $\mathbb{Z}_{p}$. Note that every minimal generating set of $A_{4}$ is of the form $\{(1,2,3),(1,2,4)\}$ or $\{(1,2,3),(1,2)(3,4)\}$ (up to automorphism), so $S$ has either 2 or 3 elements.

Case 1. Assume $\# S=2$. Write $S=\{a, b\}$.

Subcase 1.1. Assume $a=(1,2)(3,4) z$. We may assume $p=2$, for otherwise Corollary 2.11 applies with $N=\mathbb{Z}_{p}$. Let $b$ be the second element of $S$; we may assume $b$ is either $(1,2,3)$ or $(1,2,3) z$. 
- If $b=(1,2,3)$, then $\left(\left(b^{2}, a\right)^{2},\left(b^{-2}, a\right)^{2}\right)^{2}$ is a hamiltonian cycle.

- If $b=(1,2,3) z$, then $\left(b^{5}, a, b^{-5}, a\right)^{2}$ is a hamiltonian cycle.

Subcase 1.2. Assume $(1,2)(3,4) z \notin S$. We may assume $(1,2,3) z \in S$. Then we may assume $p=3$, for otherwise it is not difficult to verify that Lemma 2.14 applies with $s_{1}=(1,2,3) z$.

Let $a=(1,2,3) z$, and let $b$ be the other element of $S$. Since $\{(1,2,3) z,(1,2)(3,4)\rangle \neq$ $A_{4} \times \mathbb{Z}_{3}$, we must have $b=(1,2,4) z^{i}$ for some $i$. By applying an automorphism, we may assume $i=0$. Then a hamiltonian cycle is given by

$$
\left(a^{-2}, b^{-2}, a^{2}, b,\left(a^{-2}, b^{2}\right)^{2}, a^{2}, b, a^{-2}, b^{-2}, a, b, a^{2}, b^{-1}, a^{-2}, b^{2}, a^{-2}, b, a^{-1}, b\right) .
$$

Case 2. Assume $\# S=3$. We may assume $S \cap \mathbb{Z}_{p}=\emptyset$, for otherwise Lemma 2.27(1) applies. Then, from the minimality of $S$, it is not difficult to see that we must have $p=3$ and (after applying an automorphism) $S$ contains both $(1,2,3)$ and $(1,2,3) z$, so Corollary 2.11 applies (with $N=\mathbb{Z}_{p}$ ).

Our proof [17] of the following result consists of two pages of unilluminating case-bycase analysis, so we omit it.

Lemma 2.32 ([17]). Every connected Cayley graph on the alternating group $A_{5}$ has a hamiltonian cycle.

\section{H Some facts from group theory}

The following well-known consequence of Sylow's Theorems will be used several times.

Lemma 2.33 ([8, Thm. 25.1]). Suppose $G$ is a finite group, $p$ is a prime number, and $P$ is a Sylow p-subgroup of $G$. If 1 is the only divisor $k$ of $|G| /|P|$, such that $k \equiv 1(\bmod p)$, then $P$ is a normal subgroup of $G$.

We recall a few basic facts about the Frattini subgroup.

Definition 2.34. The Frattini subgroup of a finite group $G$ is the intersection of all the maximal subgroups of $G$. It is denoted $\Phi(G)$.

Proposition 2.35 (cf. [11, Thms. 5.1.1 and 5.1.3]). Let $S$ be a minimal generating set of a finite group $G$. Then:

1. $\Phi(G)$ is a normal subgroup of $G$.

2. $S \cap \Phi(G)=\emptyset$.

3. $S$ is a minimal generating set of $G / \Phi(G)$.

4. If $G$ is a p-group, then $\Phi(G)=[G, G] \cdot\left\langle g^{p} \mid g \in G\right\rangle$.

And we also recall some very basic facts about Hall subgroups.

Definition 2.36. A subgroup $H$ of a finite group $G$ is a Hall subgroup if $|H|$ is relatively prime to $|G| /|H|$.

Proposition 2.37 ([11, Thm. 6.4.1(i)]). Let

- $G$ be a finite group that is solvable, and

- $k$ be a divisor of $|G|$, such that $k$ is relatively prime to $|G| / k$.

Then $G$ has a Hall subgroup whose order is precisely $k$. 


\section{Groups of order $8 p$}

We begin with a special case:

Lemma 3.1. If $|G|=8 p$, where $p$ is prime, and the Sylow $p$-subgroups are not normal, then every connected Cayley graph on $G$ has a hamiltonian cycle.

Proof. Let $P$ be a Sylow $p$-subgroup of $G$. Sylow's Theorem (2.33) implies $p$ is either 3 or 7 .

Case 1. Assume $p=3$. The normalizer $N_{G}(P)$ is not all of $G$, so $\left|G: N_{G}(P)\right|=4$. Letting $G$ act on the cosets of $N_{G}(P)$ by translation yields a homomorphism from $G$ to $S_{4}$. Then either $G=S_{4}$ (so Lemma 2.30 applies), or $N_{G}(P)$ contains a normal subgroup $N$ of $G$, which must be of order 2 , and thus $N \subseteq Z(G)$.

Since $|G / N|=12$, and the Sylow 3 -subgroup is not normal, we have $G / N \cong A_{4}$. We may assume $G \nsubseteq A_{4} \times \mathbb{Z}_{2}$ (otherwise Lemma 2.31 applies), so it is easy to see that $G \cong \mathbb{Z}_{3} \ltimes Q_{8}$. Since $G / N \cong A_{4} \cong \mathbb{Z}_{3} \ltimes\left(\mathbb{Z}_{2} \times \mathbb{Z}_{2}\right)$, the proof of Corollary 2.16 tells us that $\left(s_{1}^{2}, s_{2}^{-1}, s_{1}^{-2}, s_{2}\right)^{2}$ is a hamiltonian cycle in $\operatorname{Cay}(G / N ; S)$. Its endpoint in $G$ is $\left[s_{1}, s_{2}\right]^{2}$. This generates $N$ (because the square of any element of $Q_{8}-\{ \pm 1\}$ is nontrivial), so the Factor Group Lemma (2.8) provides a hamiltonian cycle in $\operatorname{Cay}(G ; S)$.

Case 2. Assume $p=7$. It is not difficult to see that we must have $G=\mathbb{Z}_{7} \ltimes\left(\mathbb{Z}_{2}\right)^{3}$. Let $x$ and $y$ be nontrivial elements of $\mathbb{Z}_{7}$ and $\left(\mathbb{Z}_{2}\right)^{3}$, respectively. Then, up to automorphism (and replacing generators by their inverses), it is clear that every minimal generating is of the form $\left\{x, x^{i} y\right\}$ with $i \in\{0,1,2,4\}$. Furthermore, since $x \equiv\left(x^{4} y\right)^{2}\left(\bmod \left(\mathbb{Z}_{2}\right)^{3}\right)$, an automorphism carries the generating set $\left\{x, x^{4} y\right\}$ to $\left\{x, x^{2} y\right\}$; so we may assume $i \neq 4$. Here are hamiltonian cycles for the three remaining cases.

$$
\begin{aligned}
& i=0:\left(\left(x^{6}, y\right)^{2},\left(x^{-6}, y\right)^{2}\right)^{2}, \\
& i=1:\left(x^{6}, x y, x y\right)^{7}, \\
& i=2:\left(\left(x^{6}, x^{2} y\right)^{2},\left(x^{-6},\left(x^{2} y\right)^{-1}\right)^{2}\right)^{2} .
\end{aligned}
$$

Proposition 3.2 (Jungreis-Friedman [14, Thm. 8.5]). If $|G|=8 p$, where $p$ is prime, then every connected Cayley graph on $G$ has a hamiltonian cycle.

Proof. Let $S$ be a minimal generating set of $G$. We may assume $p>2$, for otherwise $|G|=16$ is a prime power, so Remark 1.3 applies. We may also assume $G$ has a normal Sylow $p$-subgroup, for otherwise Lemma 3.1 applies. Hence, $G=P_{2} \ltimes \mathbb{Z}_{p}$ where $P_{2}$ is a Sylow 2-subgroup of $G$.

We assume the commutator subgroup of $G$ is not cyclic of prime order (otherwise Theorem 2.2 applies). Hence, $P_{2}$ is nonabelian, and acts nontrivially on $\mathbb{Z}_{p}$. The only nonabelian groups of order 8 are $D_{8}$ and $Q_{8}$.

Case 1. Assume $P_{2} \cong Q_{8}$. Since Aut $\mathbb{Z}_{p}$ is cyclic, and the only nontrivial cyclic quotient of $Q_{8}$ is $\mathbb{Z}_{2}$, it must be the case that the kernel of the action of $Q_{8}$ on $\mathbb{Z}_{p}$ is a subgroup of order 4 . The subgroups of order 4 in $Q_{8}$ are cyclic, so it is easy to see that $G$ is of quaternion type. Hence, Corollary 2.25 applies.

Case 2. Assume $P_{2} \cong D_{8}$. The argument of the preceding case shows that the kernel of the action of $D_{8}$ on $\mathbb{Z}_{p}$ is of order 4 . If it is cyclic, then $G \cong D_{8 p}$, which is covered by Lemma 2.22. 
Henceforth, we assume the kernel is not cyclic, so

$$
G \cong\left\{f, x, z \mid f^{2}=x^{4}=z^{p}=1, x^{f}=x^{-1}, z^{f}=z, z^{x}=z^{-1}\right\} .
$$

Note that, by Lemma 2.27, we may assume $S$ does not contain any element of $\langle z\rangle$.

Note that:

- $x^{2}$ is an element of order 2 in $Z(G)$, so $x^{2}$ belongs to every Sylow 2-subgroup, and

- $x^{2}$ is in the Frattini subgroup of $D_{8} \cong G /\langle z\rangle$, so $x^{2}$ also belongs to every maximal subgroup that contains $\langle z\rangle$.

Since every maximal subgroup of $G$ either is a Sylow 2-subgroup or contains $\langle z\rangle$, we conclude that $x^{2} \in \Phi(G)$. Since $S$ is minimal, this tells us that $S$ is a minimal generating set of $G /\left\langle x^{2}\right\rangle$. Therefore, we may assume $S$ does not contain any element $s$ such that $s^{2}=x^{2}$. (Otherwise, Corollary 2.11 applies with $t=s^{-1}$.) This means that $S$ does not contain any element of the form $x^{ \pm 1} z^{n}$.

Then, since $S$ must generate $G /\left\langle x^{2}, z\right\rangle$, we conclude that $S$ (or $S^{-1}$ ) contains elements of the form $f x z^{\ell}$ and $f x^{2 m} z^{n}$. We may assume:

- $\ell=0$ (conjugating by a power of $z$ ),

- $m=0$ (because we can conjugate by a power of $x$ and replace $x$ with $x^{-1}$ if necessary), and

- $n=0$ (otherwise, we may apply Corollary 2.11 with $N=\langle z\rangle$ and $t^{-1}=s=f z^{n}$ ).

Thus, $S$ contains both $f$ and $f x$.

Now, in order to generate $z$, the set $S$ must contain either $x^{2} z$ or $f x^{i} z$, for some $i$ (up to replacing $z$ with one of its powers).

Subcase 2.1. Assume $x^{2} z$ in $S$. We must have $S=\left\{f, f x, x^{2} z\right\}$ and Lemma 2.6 shows that $\left(f x, f, f x, x^{2} z\right)^{2 p}$ is a hamiltonian cycle, since $f x \cdot f \cdot f x \cdot x^{2} z=f z$ is of order $2 p$, and the vertices

$$
e, f x, f x \cdot f=x^{3} \text {, and } x^{3} \cdot f x=f x^{2}
$$

are all in different right cosets of $\langle f, z\rangle$.

Subcase 2.2. Assume there is no element of the form $x^{2} z^{i}$ in $S$. Then $f x^{i} z \in S$. Note that $i$ must be odd, for otherwise $\langle z\rangle \subset\left\langle f x^{i} z\right\rangle$, so $\left\langle f x, f x^{i} z\right\rangle=G$, contradicting the minimality of $S$. Thus, we have

$$
S=\{f, f x, f x z\} \text { or } S=\left\{f, f x, f x^{3} z\right\} .
$$

In the former case, Corollary 2.11 applies (letting $s=f x, t=f x z$, and $N=\langle z\rangle$ ).

We may now assume $S=\left\{f, f x, f x^{3} z\right\}$. Because we have $G / \mathbb{Z}_{p} \cong D_{8}$, it is easy to check that

$$
\left(f x, f x^{3} z, f x, f, f x^{3} z, f x, f x^{3} z, f\right)
$$

is a hamiltonian cycle in $\operatorname{Cay}\left(G / \mathbb{Z}_{p} ; S\right)$, and we have

$$
(f x)\left(f x^{3} z\right)(f x)(f)\left(f x^{3} z\right)(f x)\left(f x^{3} z\right)(f)=z^{3} .
$$

If $p \geq 5$, this product generates $\mathbb{Z}_{p}$, so the Factor Group Lemma (2.8) tells us there is a hamiltonian cycle in $\operatorname{Cay}(G ; S)$. 
If $p=3$, a hamiltonian cycle is given by

$$
\begin{aligned}
& \left((f, f x)^{2}, f x^{3} z, f, f x, f, f x^{3} z,\right. \\
& \left.\quad(f x, f)^{2}, f x^{3} z, f, f x^{3} z, f x, f, f x^{3} z, f, f x, f x^{3} z, f, f x^{3} z\right) .
\end{aligned}
$$

\section{Groups of order $3 p^{2}$}

Proposition 4.1. If $|G|=3 p^{2}$, where $p$ is prime, then every connected Cayley graph on $G$ has a hamiltonian cycle.

Proof. Let $S$ be a minimal generating set of $G$, and let $P$ be a Sylow $p$-subgroup of $G$. We may assume $p \geq 5$, for otherwise either $|G|=12$ is of the form $4 p$, so Corollary 2.17 applies, or $|G|=3^{3}$ is a prime power, so Remark 1.3 applies. Hence, Sylow's Theorem (2.33) tells us $P \triangleleft G$. Note that $G / P \cong \mathbb{Z}_{3}$ is abelian, so $[G, G] \subset P$. So we may assume $P \cong \mathbb{Z}_{p} \times \mathbb{Z}_{p}$, for otherwise Theorem 2.2 applies. Thus, we may assume $G=$ $\mathbb{Z}_{3} \ltimes\left(\mathbb{Z}_{p} \times \mathbb{Z}_{p}\right)$, and $[G, G]=\mathbb{Z}_{p} \times \mathbb{Z}_{p}$.

Case 1. Assume $|S|=3$. Write $S=\{s, t, u\}$. We may assume $s \notin \mathbb{Z}_{p} \times \mathbb{Z}_{p}$, so $|s|=3$. Because $S$ is minimal, we see that $\langle s, t\rangle$ and $\langle s, u\rangle$ each have order $3 p$. Let $N$ be the unique subgroup of order $p$ in $\langle s, t\rangle$, and note that $N$ is normal in $G$ (because it is normalized both by $s$ and by the abelian group $\mathbb{Z}_{p} \times \mathbb{Z}_{p}$ ).

- If $t \in N$, then Theorem 2.28 applies.

- If $t \notin N$, then we may assume $s \equiv t(\bmod N)$ (by replacing $t$ with $t^{-1}$, if necessary). So Corollary 2.11 applies.

Case 2. Assume $|S|=2$. Write $S=\{s, t\}$.

If $s$ and $t$ both have order 3 , then we may assume $s \equiv t\left(\bmod \mathbb{Z}_{p} \times \mathbb{Z}_{p}\right)$ (by replacing $t$ with $t^{-1}$, if necessary). Then $s t^{-1}$ is contained in the normal $p$-subgroup $\mathbb{Z}_{p} \times \mathbb{Z}_{p}$, so Corollary 2.4 applies.

We may now assume $|s|=3$ and $|t|=p$.

Let us determine the action of $s$ on $\mathbb{Z}_{p} \times \mathbb{Z}_{p}$.

- Define a linear transformation $T$ on $\mathbb{Z}_{p} \times \mathbb{Z}_{p}$ by $T(v)=s^{-1} v s$,

- let $m(x)$ be the minimal polynomial of $T$, and

- let $u=T(t)=s^{-1} t s$.

Note that:

- Because $|s|=3$, we know $T^{3}=I$, so $m(x)$ divides $x^{3}-1=(x-1)\left(x^{2}+x+1\right)$.

- Since $|[G, G]|=p^{2}$, we know that 1 is not an eigenvalue of $T$.

- Because $\langle s, t\rangle=G$, we know $u=T(t) \notin\langle t\rangle$, so the minimal polynomial of $T$ has degree 2 (and $\{t, u\}$ is a basis of $\mathbb{Z}_{p} \times \mathbb{Z}_{p}$ ).

We conclude that the minimal polynomial of $T$ is $x^{2}+x+1$. Thus, with respect to the basis $\{t, u\}$ of $\mathbb{Z}_{p} \times \mathbb{Z}_{p}$, we have $T(i, j)=(-j, i-j)$. In other words, $s^{-1}\left(t^{i} u^{j}\right) s=t^{-j} u^{i-j}$, so

$$
t^{i} u^{j} s=s\left(s^{-1}\left(t^{i} u^{j}\right) s\right)=s\left(t^{-j} u^{i-j}\right) \in\langle s\rangle t^{-j} u^{i-j} .
$$

The quotient multigraph $\langle s\rangle \backslash \operatorname{Cay}(G ; S)$ has the following properties: 
- it has $p^{2}$ vertices;

- it is regular of valency 4 ;

- the vertices are the ordered pairs $(i, j)$, where

$$
-\frac{p-1}{2} \leq i, j \leq \frac{p-1}{2}
$$

and

- the vertex $(i, j)$ is adjacent to:

$$
(i+1, j) ;(i-1, j) ;(-j, i-j) ; \text { and }(j-i,-i),
$$

where calculations are performed modulo $p$ (see 4.2).

Furthermore, we observe that for any prime $p$, there are precisely two multiple edges:

- If $p=3 k+1$, then, with $j=k$ and $i=2 j$, we have

$$
(-j, i-j)=(-k, k)=(2 k+1, k)=(i+1, j),
$$

and, with $j=-k$ and $i=2 j$, we have

$$
(-j, i-j)=(k,-k)=(-2 k-1,-k)=(i-1, j) .
$$

- If $p=3 k+2$, then, with $j=k+1$ and $i=2 j$, we have

$$
(-j, i-j)=(-k-1, k+1)=(2 k+1, k+1)=(i-1, j),
$$

and, with $j=-(k+1)$ and $i=2 j$, we have

$$
(-j, i-j)=(k+1,-(k+1))=(-2 k-1,-(k+1))=(i+1, j) .
$$

We now construct a hamiltonian cycle in the multigraph. Beginning at the vertex $(1,1)$, we proceed along the following sequence of edges (where $\{n\}$ denotes the remainder when $n$ is divided by $p$, so $0 \leq\{n\}<p$ ):

$$
\begin{gathered}
\left(\left[\left(t^{-1}\right)^{\{3 j-1\}}, s^{-1}, t^{\{-3 j-1\}}, s^{-1}\right]_{j=1}^{(p-3) / 2},\right. \\
{\left[\left(t^{-1}\right)^{(p-5) / 2}, s^{-1}, t^{(p+1) / 2}, s\right]} \\
{\left[t^{p-1}, s^{-1}\right],} \\
{\left[t^{\{3 j-1\}}, s,\left(t^{-1}\right)^{\{-3 j-1\}}, s^{*}\right]_{j=-(p-1) / 2}^{-k-1}} \\
{\left[\left(t^{-1}\right)^{\{3 j-1\}}, s^{-1}, t^{\{-3 j-1\}}, s^{-1}\right]_{j=-k}^{-2},} \\
\left.\left[\left(t^{-1}\right)^{p-4}, s^{-1}, t^{2}, s\right]\right) .
\end{gathered}
$$

Here $s^{*}$ indicates $s$ unless $j=-k-1$ and $p=3 k+1$, in which case it indicates $s^{-1}$.

We make a few observations that will aid the reader in verifying that this is indeed a hamiltonian cycle. We will see that each portion enclosed within square brackets traverses 


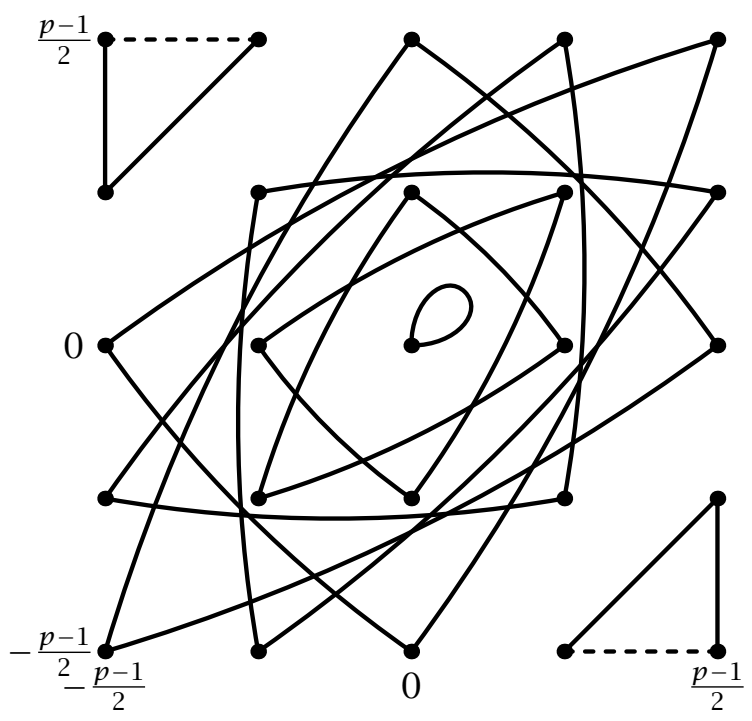

Figure 2: The $s$-edges in the multigraph when $p=5$. The $t$-edges (not drawn) are horizontal. Thus, there are two double edges (dashed, at top left and bottom right) in the multigraph.

all of the vertices in some row of the multigraph, and passes on to the next row; the parameter $j$ represents the row being traversed. For convenience, we will use $\bar{n}$ to denote the integer congruent to $n$ modulo $p$ that is between $-(p-1) / 2$ and $(p-1) / 2$ (inclusive).

In the first portion enclosed within square brackets, we begin each row at a vertex of the form $(\overline{2 j-1}, j)$. The $t^{-1}$ edges cover every vertex between this and $(-j, j)$, moving leftwards; $s^{-1}$ takes us to the vertex $(\overline{2 j}, j)$, and the $t$ edges cover every vertex between this and $(\overline{-j-1}, j)$, completing the row. The $s^{-1}$ edge then takes us to $(\overline{2 j+1}, j+1)$, which has the required form to continue this pattern. In this way, we cover all of the vertices from rows 1 through $(p-3) / 2$.

Then, the second portion enclosed within square brackets covers the vertices of row $(p-1) / 2$ in the same way, except that it ends with an $s$-edge, taking us from $((p-$ $1) / 2,(p-1) / 2)$ to $(-(p-1) / 2,0)$.

The third portion enclosed within square brackets takes us through the vertices of row 0 , ending at $((p-1) / 2,0)$, and to vertex $(-(p-1) / 2,-(p-1) / 2)$, which has the form $(\overline{-j+1}, j)$.

In the fourth portion enclosed within square brackets, we use the same pattern on rows $-(p-1) / 2$ through $-k-1$ that we used on rows $k+1$ through $(p-1) / 2$, rotated by 180 degrees. So in each row we begin at a vertex of the form $(\overline{-j+1}, j)$, move right until we reach $(\overline{2 j}, j)$, then jump to $(-j, j)$, move left to the vertex $(\overline{2 j+1}, j)$ (completing the row), and pass to vertex $(-j, j+1)$ in the next row, which again has the required form to continue. The $s^{*}$ edge ends this portion at the vertex $(k+1,-k)$ if $p=3 k+2$, and at $(k, \overline{2 k+1})$ if $p=3 k+1$; both of these are equal to $(\overline{-2 k-1},-k)$. This has the form $(\overline{2 j-1}, j)$ again. 
Now the fifth portion uses the same pattern we began with, to cover the vertices in rows $-k$ through -2 .

The final portion covers row -1 , using the same pattern, finishing at the vertex $(0,-1)$. Now edge $s$ takes us to $(1,1)$, completing the hamiltonian cycle.

Notice that in each row (except row 0), this cycle traversed the unique $s$-edge whose endpoints were both in that row. In particular, this cycle traversed each of the multi-edges noted above. So Corollary 2.9 implies there is a hamiltonian cycle in $\operatorname{Cay}(G ; S)$.

\section{Groups of order $4 p^{2}$}

We begin with a special case.

Lemma 5.1 (Jungreis-Friedman [14, Thm. 7.3]). If $p$ is prime, then every connected Cayley graph on $D_{2 p} \times D_{2 p}$ has a hamiltonian cycle.

Proof. Let $S$ be a minimal generating set of $G=D_{2 p} \times D_{2 p}$, where $p$ is prime. We may assume $p \geq 3$, for otherwise $|G|=16$ is a power of 2 , so Remark 1.3 applies.

Notice that the elements of $S$ cannot consist exclusively of pairs of nontrivial reflections, together with pairs of (possibly trivial) rotations, since such a set cannot generate the element $(f, e)$ of $D_{2 p} \times D_{2 p}$ (for any reflection $f$ ). This is because getting $f$ in the first coordinate requires taking the product of an odd number of elements that are pairs of nontrivial reflections, while getting $e$ in the second coordinate requires taking the product of an even number of such elements. Therefore, as $S$ must contain an element with a reflection in its first coordinate, we may assume that either $(f, e) \in S$ or $\left(f, x^{\prime}\right) \in S$, for some reflection $f$ and some nontrivial rotation $x^{\prime}$.

Case 1. Assume $|S|=2$. In order to generate the entire group, $S$ must include either two reflections, or a reflection and a rotation, within each of the dihedral factors. Up to automorphism, there are only two generating sets that satisfy this condition: $\left\{\left(x, f^{\prime}\right),\left(f, x^{\prime}\right)\right\}$, or $\left\{\left(f_{1}, x^{\prime}\right),\left(f_{2}, f^{\prime}\right)\right\}$, where $x, x^{\prime}$ are nontrivial rotations and $f, f^{\prime}, f_{1}, f_{2}$ are reflections and $f_{1} \neq f_{2}$.

We will now verify that each of the above generating sets satisfies the conditions of Lemma 2.14, so that by that lemma, $\operatorname{Cay}(G ; S)$ does indeed have a hamiltonian cycle.

Subcase 1.1. Assume $s_{1}=\left(x, f^{\prime}\right)$ and $s_{2}=\left(f, x^{\prime}\right)$. Letting $\gamma=\left[s_{1}, s_{2}\right]$, we have

$$
\gamma=\left(x^{-1} f x f, f^{\prime}\left(x^{\prime}\right)^{-1} f^{\prime} x^{\prime}\right)=\left(x^{-2},\left(x^{\prime}\right)^{2}\right) .
$$

So

- $2\left|s_{1}\right||\gamma|=2(2 p)(p)=4 p^{2}=|G|$.

- $\left\langle s_{1}\right\rangle\langle\gamma\rangle=\left\{\left(x^{i} x^{-2 j},\left(f^{\prime}\right)^{i}\left(x^{\prime}\right)^{2 j}\right) \mid i, j \in \mathbb{Z}\right\}$, which never has $f$ in the first coordinate, so $s_{2}$ is not in this set.

- If $\gamma^{i}=s_{1}^{j}$ for some $i, j$, then $\left(x^{\prime}\right)^{2 i}=\left(f^{\prime}\right)^{j}$, so we must have $\left(x^{\prime}\right)^{2 i}=\left(f^{\prime}\right)^{j}=e$, so $i \equiv 0(\bmod p)$. But then $x^{-2 i}=e$, so $\gamma^{i}=(e, e)$. Therefore, $\langle\gamma\rangle \cap\left\langle s_{1}\right\rangle=\{e\}$.

- If $\gamma^{i}=s_{2}^{-1} s_{1}^{j} s_{2}$ for some $i, j$, then $\left(x^{\prime}\right)^{2 i}=\left(x^{\prime}\right)^{-1}\left(f^{\prime}\right)^{j} x^{\prime}$, so $\left(x^{\prime}\right)^{2 i}=e=\left(f^{\prime}\right)^{j}$, so $i \equiv 0(\bmod p)$. But then $x^{-2 i}=e$, so $\gamma^{i}=(e, e)$. Therefore, $\langle\gamma\rangle \cap\left(s_{2}^{-1}\left\langle s_{1}\right\rangle s_{2}\right)=$ $\{e\}$. 
Subcase 1.2. Assume $s_{1}=\left(f_{1}, x^{\prime}\right)$ and $s_{2}=\left(f_{2}, f^{\prime}\right)$, with $f_{1} \neq f_{2}$. We may assume $f_{2}=f_{1} x$. Then with $\gamma=\left[s_{1}, s_{2}\right]$, we have

$$
\gamma=\left(f_{1}\left(f_{1} x\right) f_{1}\left(f_{1} x\right),\left(x^{\prime}\right)^{-1} f^{\prime} x^{\prime} f^{\prime}\right)=\left(x^{2},\left(x^{\prime}\right)^{-2}\right) .
$$

So

- $2\left|s_{1}\right||\gamma|=2(2 p)(p)=4 p^{2}=|G|$;

- $\left\langle s_{1}\right\rangle\langle\gamma\rangle=\left\{\left(f_{1}^{i} x^{2 j},\left(x^{\prime}\right)^{i}\left(x^{\prime}\right)^{-2 j}\right) \mid i, j \in \mathbb{Z}\right\}$, which never has $f^{\prime}$ in the second coordinate, so $s_{2}$ is not in this set.

- If $\gamma^{i}=s_{1}^{j}$ for some $i, j$, then $x^{2 i}=f_{1}^{j}$, so we must have $x^{2 i}=e$, so $i \equiv 0(\bmod p)$. But then $\left(x^{\prime}\right)^{-2 i}=e$, so $\gamma^{i}=(e, e)$. Therefore, $\langle\gamma\rangle \cap\left\langle s_{1}\right\rangle=\{e\}$.

- If $\gamma^{i}=s_{2}^{-1} s_{1}^{j} s_{2}$ for some $i, j$, then $x^{2 i}=f_{2} f_{1}^{j} f_{2}$, so $x^{2 i}=e$, so $i \equiv 0(\bmod p)$. But then $\left(x^{\prime}\right)^{-2 i}=e$, so $\gamma^{i}=(e, e)$. Therefore, $\langle\gamma\rangle \cap\left(s_{2}^{-1}\left\langle s_{1}\right\rangle s_{2}\right)=\{e\}$.

Case 2. Assume $|S|=3$. In what follows, we will be applying Corollary 2.19 repeatedly. We will verify some of its conditions here, so that only one condition will need to be checked each time we use it below. Namely, we already know that $S$ is minimal and $|S| \geq 3$. Furthermore, we assume, by induction on $|G|$, that Cay $\left(\left\langle S-\left\{s_{1}\right\}\right\rangle ; S-\left\{s_{1}\right\}\right)$ has a hamiltonian cycle, for any $s_{1} \in S$. Thus, in order to apply the corollary, all that remains is to verify that there exist two distinct generators $s_{1}, s_{2} \in S$ such that $\left|s_{1} s_{2}\right|=$ $|G| /\left|\left\langle S-\left\{s_{1}\right\}\right\rangle\right|$ is prime.

Subcase 2.1. Assume $S$ is disjoint from $D_{2 p} \times\{e\}$ and $\{e\} \times D_{2 p}$. The discussion preceding Case 1 implies that we may assume $s=\left(f, x^{\prime}\right) \in S$, for some reflection $f$ and some nontrivial rotation $x^{\prime}$.

The generating set $S$ must also be an element whose second coordinate is a reflection. By the assumption of this subcase, the first coordinate cannot be trivial. And it also cannot be a nontrivial rotation (because $S$ is minimal and $|S|=3$ ). Therefore, it is a reflection. From the minimality of $S$, we conclude that it is $f$ : that is, $s_{1}=\left(f, f^{\prime}\right) \in S$, for some reflection $f^{\prime}$.

Now, let $s_{2}=\left(y, y^{\prime}\right)$ be the third element of $S$. To generate $G$, we must have $y \neq f$. (And $y^{\prime}$ is nontrivial, from the assumption of this subcase.) Note that if $y$ is a reflection, then either $\left\langle s_{2}, s\right\rangle=G$ or $\left\langle s_{2}, s_{1}\right\rangle=G$, depending on whether $y^{\prime}$ is a rotation or a reflection. Thus, the minimality of $S$ implies that $y$ is a rotation $x$. Then, since $\left\langle s,\left(x, f^{\prime \prime}\right)\right\rangle=G$ for any rotation $f^{\prime \prime}$, the minimality of $S$ implies that $y^{\prime}$ is a rotation. Thus, $s_{2}=\left(x, x^{\prime \prime}\right) \in S$, for some nontrivial rotations $x$ and $x^{\prime \prime}$. Then $\left|s_{1} s_{2}\right|=\left|\left(f x, f^{\prime} x^{\prime \prime}\right)\right|=2$ and $\left\langle S-\left\{s_{1}\right\}\right\rangle=$ $D_{2 p} \times \mathbb{Z}_{p}$ has index 2 , so Corollary 2.19 provides a hamiltonian cycle in Cay $(G ; S)$.

Subcase 2.2. Assume $(f, e) \in S$. As there must be a reflection in the second coordinate of some element of $S$, we have either $\left(x, f^{\prime}\right) \in S$, or $\left(e, f^{\prime}\right) \in S$, or $\left(f_{1}, f^{\prime}\right) \in S$.

Subsubcase 2.2.1. Assume $\left(x, f^{\prime}\right) \in S$, with $|x|=p$. Then $\left(x, f^{\prime}\right)$ generates a subgroup of $G$ of order $2 p$. Let $s_{1}=(f, e)$. Since

$$
\left[s_{1}, G\right]=\langle(x, e)\rangle \subset\left\langle\left(x, f^{\prime}\right)\right\rangle \subset\left\langle S-\left\{s_{1}\right\}\right\rangle,
$$

we know that $s_{1}$ normalizes $\left\langle S-\left\{s_{1}\right\}\right\rangle$, so $G=\left\langle s_{1}\right\rangle\left\langle S-\left\{s_{1}\right\}\right\rangle$. Furthermore, because $S$ is a minimal generating set, we know $s_{1} \notin\left\langle S-\left\{s_{1}\right\}\right\rangle$. Since $\left|s_{1}\right|=2$ is prime, this implies 
$\left\langle s_{1}\right\rangle \cap\left\langle S-\left\{s_{1}\right\}\right\rangle=\{e\}$, so $|G|=\left|\left\langle s_{1}\right\rangle\right| \cdot\left|\left\langle S-\left\{s_{1}\right\}\right\rangle\right|$. Therefore $|G| /\left|\left\langle S-\left\{s_{1}\right\}\right\rangle\right|=2$. Also, with $s_{2}=\left(x, f^{\prime}\right)$, we have $\left|s_{1} s_{2}\right|=2$. So Corollary 2.19 tells us that $\operatorname{Cay}(G ; S)$ is hamiltonian.

Subsubcase 2.2.2. Assume either $\left(e, f^{\prime}\right) \in S$, or $\left(f, f^{\prime}\right) \in S$. Let $r_{1}=(f, e)$ and let $r_{2}$ be either $\left(e, f^{\prime}\right)$ or $\left(f, f^{\prime}\right)$, the other element that we know to be in $S$. Note that, because $\left|r_{1} r_{2}\right|=2$,

if either $\left\langle S-\left\{r_{1}\right\}\right\rangle$ or $\left\langle S-\left\{r_{2}\right\}\right\rangle$ has index 2 in $G$, then Corollary 2.19 tells us $\operatorname{Cay}(G ; S)$ is hamiltonian.

We claim that we may assume no element of $S$ consists of a nontrivial rotation in one coordinate together with a reflection in the other. To see this, observe, first of all, that we are not in Subsubcase 2.2.1, so the reflection cannot be in the second coordinate. Thus, we suppose $S$ contains some $\left(f_{1}, x^{\prime}\right)$. Since $S$ is minimal and contains $(f, e)$, we must have $f_{1} \neq f$. Therefore $\left\langle\left(f_{1}, x^{\prime}\right),\left(f, f^{\prime}\right)\right\rangle=G$, so combining the minimality of $S$ with the definition of $r_{2}$ tells us that $r_{2}=\left(e, f^{\prime}\right)$. Thus, Subsubcase 2.2.1 applies, after interchanging the two factors of $G$.

If the third element of $S$ is of the form $\left(x, x^{\prime}\right)$, then $\left\langle S-\left\{r_{2}\right\}\right\rangle$ has index 2 in $G$, so $\operatorname{Cay}(G ; S)$ has a hamiltonian cycle by (5.2). Thus, from the preceding paragraph, we may assume that the third element of $S$ is of the form $\left(f_{1}, e\right),\left(e, f_{1}^{\prime}\right)$, or $\left(f_{1}, f_{1}^{\prime}\right)$. However, only the last of these can generate all of $G$ when combined with $r_{1}$ and $r_{2}$. Therefore

$$
S=\left\{r_{1}, r_{2},\left(f_{1}, f_{1}^{\prime}\right)\right\}
$$

Furthermore, we must have $f_{1} \neq f$ and $f_{1}^{\prime} \neq f^{\prime}$.

If $r_{2}=\left(f, f^{\prime}\right)$, then $\left|r_{2}\left(f_{1}, f_{1}^{\prime}\right)\right|=p$ and $\left|\left\langle r_{1},\left(f_{1}, f_{1}^{\prime}\right)\right\rangle\right|=4 p$, so Corollary 2.19 applies. If $r_{2}=\left(e, f^{\prime}\right)$ then Corollary 2.19 cannot be applied, but we claim that

$$
\left(\left(\left(f_{1}, f_{1}^{\prime}\right),(f, e)\right)^{p} \#,\left(e, f^{\prime}\right)\right)^{2 p}
$$

is a hamiltonian cycle. To verify this, we first calculate that:

- $\left(\left(f_{1} f\right)^{p} f^{-1},\left(f_{1}^{\prime}\right)^{p} f^{\prime}\right)=\left(f, f_{1}^{\prime} f^{\prime}\right)$,

- $\left(\left(f_{1} f\right)^{i},\left(f_{1}^{\prime}\right)^{i}\right) \in\left\langle\left(f, f_{1}^{\prime} f^{\prime}\right)\right\rangle \cdot \begin{cases}\left(\left(f_{1} f\right)^{i}, e\right) & \text { if } i \text { is even, } \\ \left(\left(f_{1} f\right)^{i}, f_{1}^{\prime}\right) & \text { if } i \text { is odd }\end{cases}$

- $\left(\left(f_{1} f\right)^{i},\left(f_{1}^{\prime}\right)^{i}\right) \cdot\left(f_{1}, f_{1}^{\prime}\right) \in\left\langle\left(f, f_{1}^{\prime} f^{\prime}\right)\right\rangle \cdot \begin{cases}\left(\left(f_{1} f\right)^{-(i+1)}, f_{1}^{\prime}\right) & \text { if } i \text { is even, } \\ \left(\left(f_{1} f\right)^{-(i+1)}, e\right) & \text { if } i \text { is odd. }\end{cases}$

The conclusion that we have a hamiltonian cycle now follows easily from Lemma 2.6.

Subsubcase 2.2.3. Assume $\left(f_{1}, f^{\prime}\right) \in S$ with $f_{1} \neq f$.

If the third element of $S$ is of the form $\left(f_{2}, f_{1}^{\prime}\right)$, then $f_{1}^{\prime} \neq f^{\prime}$ (otherwise $S$ would not generate $G$ ). Because $\left|\left(f_{2}, f_{1}^{\prime}\right)\left(f_{1}, f^{\prime}\right)\right|=p$ and $\left|\left\langle(f, e),\left(f_{1}, f^{\prime}\right)\right\rangle\right|=4 p$, the conditions of Corollary 2.19 are satisfied with $s_{1}=\left(f_{2}, f_{1}^{\prime}\right)$.

If the third element of $S$ is of the form $\left(f_{2}, x^{\prime}\right)$, then $x^{\prime}$ must be nontrivial (or else $S$ would not generate $G$ ), so $f_{2}=f_{1}$; otherwise, $(f, e)$ is redundant. Then, since $\mid\left(f_{1}, x^{\prime}\right)$ $(f, e) \mid=p$ and $\left|\left\langle(f, e),\left(f_{1}, f^{\prime}\right)\right\rangle\right|=4 p$, the conditions of Corollary 2.19 are satisfied. 
If the third element of $S$ is of the form $\left(e, x^{\prime}\right)$, then, since $\left|x^{\prime}\right|=p$, Lemma 2.27 provides a hamiltonian cycle in $\operatorname{Cay}(G ; S)$.

Finally, since we are not in Subsubcase 2.2.1 or 2.2.2, we may now assume the third element of $S$ is of the form $\left(x, x^{\prime}\right)$, with $x$ and $x^{\prime}$ nontrivial. Let $s_{1}=(f, e)$ and $s_{2}=$ $\left(f_{1}, f^{\prime}\right)$. We have $\left|s_{1} s_{2}\right|=2 p$, and $\left|\left\langle\left(x, x^{\prime}\right),\left(f_{1}, f^{\prime}\right)\right\rangle\right|=2 p$. Furthermore, $\left\langle s_{1} s_{2}\right\rangle=$ $\left\langle(x, e),\left(e, f^{\prime}\right)\right\rangle$. The intersection of this group with $\left\langle\left(x, x^{\prime}\right),\left(f_{1}, f^{\prime}\right)\right\rangle$ is clearly trivial, so Lemma 2.18 applies.

Case 3. Assume $|S|=4$. Because $S$ generates $G /\left(\mathbb{Z}_{p} \times \mathbb{Z}_{p}\right)$, we know that it contains an element of the form $(f, e)$ or $\left(e, f^{\prime}\right)$. Let us assume $(f, e) \in S$.

Subcase 3.1. Assume $S \cap\left(\mathbb{Z}_{p} \times \mathbb{Z}_{p}\right) \neq \emptyset$. Let $\left(x, x^{\prime}\right) \in S \cap\left(\mathbb{Z}_{p} \times \mathbb{Z}_{p}\right)$. We may assume that $x$ and $x^{\prime}$ are both nontrivial, for otherwise $\left\langle\left(x, x^{\prime}\right)\right\rangle \triangleleft G$, so Lemma 2.27 applies. But then $\left\langle(f, e),\left(x, x^{\prime}\right)\right\rangle$ has index 2 in $G$, which contradicts the fact that $|S|=4$.

Subcase 3.2. Assume $S \cap\left(\mathbb{Z}_{p} \times \mathbb{Z}_{p}\right)=\emptyset$. We may assume $S$ contains an element of the form $\left(f_{1}, f^{\prime}\right)$ with $f_{1}$ and $f^{\prime}$ nontrivial, for otherwise $\operatorname{Cay}(G ; S)$ is isomorphic to a Cartesian product Cay $\left(D_{2 p} ; S_{1}\right) \times \operatorname{Cay}\left(D_{2 p} ; S_{1}^{\prime}\right)$, and the Cartesian product of hamiltonian graphs is hamiltonian.

We must have $f_{1}=f$, for otherwise $\left\langle(f, e),\left(f_{1}, f^{\prime}\right)\right\rangle$ has index $p$ in $G$, contradicting the fact that $|S|=4$. So $\left(f, f^{\prime}\right) \in S$.

In order to generate $D_{2 p} \times\{e\}, S$ must contain an element whose first coordinate is not $f$. The second coordinate must be trivial (for otherwise combining it with $(f, e)$ generates a subgroup of index $p$ ). That is, we have $\left(f_{2}, e\right) \in S$, with $f_{2} \neq f$. But then $\left\langle\left(f_{2}, e\right),\left(f, f^{\prime}\right)\right\rangle$ generates a subgroup of index $p$, contradicting the fact that $|S|=4$.

Proposition 5.3. If $|G|=4 p^{2}$, where $p$ is a prime, then every connected Cayley graph on $G$ has a hamiltonian cycle.

Proof. Let $S$ be a minimal generating set of $G$. Clearly we can assume that $p \geq 3$, for otherwise $|G|$ is a prime power, so Remark 1.3 applies. Let $P$ denote a Sylow $p$-subgroup of $G$, and let $P_{2}$ be a Sylow 2-subgroup.

Case 1. Assume $P \Varangle G$. Then by Sylow's theorem (2.33) we have $p=3$ and $\mid G$ : $N_{G}(P) \mid=4$, so $N_{G}(P)=P$. Since $P$ is Abelian we thus have $P=Z\left(N_{G}(P)\right)$ and Burnside's theorem on normal $p$-complements [11, Thm. 7.4.3] implies that $P_{2} \triangleleft G$. Since $[G, G] \leq P_{2}$ we can assume $[G, G]=P_{2} \cong \mathbb{Z}_{2} \times \mathbb{Z}_{2}$ for otherwise Theorem 2.2 applies. The kernel of the action of $P$ on $\mathbb{Z}_{2} \times \mathbb{Z}_{2}$ is thus of order 3 and the only groups to consider are $\mathbb{Z}_{9} \ltimes\left(\mathbb{Z}_{2} \times \mathbb{Z}_{2}\right)$ and $A_{4} \times \mathbb{Z}_{3}$, which are covered in Corollary 2.16 and Lemma 2.31, respectively.

Case 2. Assume $P \triangleleft G$. Since $P_{2}$ is Abelian we have $[G, G] \leq P$ and we can assume $[G, G]=P \cong \mathbb{Z}_{p} \times \mathbb{Z}_{p}$ for otherwise Theorem 2.2 applies.

If $P_{2} \cong \mathbb{Z}_{2} \times \mathbb{Z}_{2}$ then either there is a nonidentity element of $P_{2}$ acting trivially on $P$, in which case $G$ is of dihedral type and Proposition 2.23 applies, or all three nonidentity elements of $P_{2}$ act nontrivially on $P$ and $G \cong D_{2 p} \times D_{2 p}$ which is covered by Lemma 5.1.

We can thus assume that $P_{2} \cong \mathbb{Z}_{4}$. Denote a generator of $\mathbb{Z}_{4}$ by $f$ and the generators of $\mathbb{Z}_{p} \times \mathbb{Z}_{p}$ by $x$ and $y$. Note that since $[G, G]=P$, we know $f$ cannot act trivially on any nonidentity element of $P$. 
Because $S$ generates $G /\left(\mathbb{Z}_{p} \times \mathbb{Z}_{p}\right)$, it has to contain an element of the form $f x^{i} y^{j}$ or its inverse. Conjugating by an appropriate power of $x$ and $y$, we can thus assume

$$
f \in S \text {. }
$$

Also, in order to generate $P$, the set $S$ must have at least one element of the form $f^{i} x^{j} y^{k}$, with either $j$ or $k$ nonzero.

Also, since $\left(f^{2}\right)^{2}=e$, we know that 1 and -1 are the only possible eigenvalues of the linear transformation defined by $f^{2}$ on the vector space $\mathbb{Z}_{p} \times \mathbb{Z}_{p}$. Thus, by choosing $x$ and $y$ to be eigenvectors of $f^{2}$, we may assume $x^{f^{2}} \in\left\{x^{ \pm 1}\right\}$ and $y^{f^{2}} \in\left\{y^{ \pm 1}\right\}$.

Subcase 2.1. Assume $f^{2}$ acts trivially on $\mathbb{Z}_{p} \times \mathbb{Z}_{p}$. This means that $f$ acts by an automorphism of order 2 . Since the automorphism does not fix any nontrivial element of $\mathbb{Z}_{p} \times \mathbb{Z}_{p}$, this implies that $g^{f}=g^{-1}$ for all $g \in \mathbb{Z}_{p} \times \mathbb{Z}_{p}$. Hence $G$ is of quaternion type, so Corollary 2.25 applies.

Subcase 2.2. Assume $|S|=2$ (and $f^{2}$ is nontrivial on $\mathbb{Z}_{p} \times \mathbb{Z}_{p}$ ). Write $S=\left\{f, f^{k} z\right\}$, with $z \in\langle x, y\rangle$. We may assume $0 \leq k \leq 2$. Note that we must have $z^{f} \notin\langle z\rangle$, because $S$ generates $G$.

Subsubcase 2.2.1. Assume $k=0$. Note that, since $[z, f]$ is not in $\langle z\rangle$ or $\langle z\rangle^{f}$,

- every element of $\mathbb{Z}_{p} \times \mathbb{Z}_{p}$ has a unique representation of the form $[z, f]^{i} z^{j}$ with $0 \leq i, j<p-1$, and

- every element of $f^{-1}\langle z\rangle$ has a unique representation of the form $[z, f]^{i} z^{-1} f^{-1} z^{-j}$ with $0 \leq i, j<p-1$.

Therefore the vertices visited by the path $\left(z^{p-1}, f^{-1}, z^{-(p-1)}, f\right)^{p} \#$ are all in different right cosets of $\left\langle f^{-2}\right\rangle$, so Lemma 2.6 tells us that

$$
\left(\left(z^{p-1}, f^{-1}, z^{-(p-1)}, f\right)^{p} \#, f^{-1}\right)^{2}
$$

is a hamiltonian cycle in $\operatorname{Cay}(G ; S)$.

Subsubcase 2.2.2. Assume $k=1$. Note that $\left(\left(f^{3}, f z\right)^{p-1}, f^{-3}\right)$ is a hamiltonian path in the subgraph of $\operatorname{Cay}(G ;\{f, f z\})$ induced by $\langle z\rangle\langle f\rangle$. Letting $g=z^{-1}\left(z^{-1}\right)^{f^{-1}}$, we have $g \notin\langle z\rangle$, so the vertices in the path are all in different right cosets of $\langle g\rangle$. Therefore, Lemma 2.6 tells us that

$$
\left(\left(f^{3}, f z\right)^{p-1}, f^{-3},(f z)^{-1}\right)^{p}
$$

is a hamiltonian cycle in $\operatorname{Cay}(G ; S)$.

Subsubcase 2.2.3. Assume $k=2$.

Subsubsubcase 2.2.3.1. Assume $f^{2}$ does not invert $\mathbb{Z}_{p} \times \mathbb{Z}_{p}$. We may assume $x^{f^{2}}=x^{-1}, y^{f^{2}}=y$, and $z=x y$. Corollary 2.11 applies, because $\left(f^{2} x y\right)^{2}=y^{2} \in\langle y\rangle \triangleleft G$.

Subsubsubcase 2.2.3.2. Assume $f^{2}$ inverts $\mathbb{Z}_{p} \times \mathbb{Z}_{p}$. We claim that

$$
\left(\left(\left(f^{2} z, f^{-1}\right)^{2},\left(f^{2} z, f\right)^{2}\right)^{(p-1) / 2}, f^{2} z, f^{-1}, f^{2} z, f\right)^{p}
$$

is a hamiltonian cycle. 
To see this, we first calculate the product

$$
\left(\left(\left(f^{2} z\right)\left(f^{-1}\right)\right)^{2}\left(\left(f^{2} z\right)(f)\right)^{2}\right)^{(p-1) / 2}\left(f^{2} z\right)\left(f^{-1}\right)\left(f^{2} z\right)(f)=z^{-1}
$$

Now, we calculate the vertices $\left(g_{0}, g_{1}, g_{2}, \ldots, g_{4 p-1}\right)$ visited by the walk

$$
\left(\left(\left(f^{2} z, f^{-1}\right)^{2},\left(f^{2} z, f\right)^{2}\right)^{(p-1) / 2}, f^{2} z, f^{-1}, f^{2} z\right)
$$

in the quotient graph $\langle z\rangle \backslash \operatorname{Cay}(G ; S)$. Letting $y=z^{f}$, and noting that each vertex of the quotient has a unique representative in $\langle y\rangle\langle f\rangle$, we calculate that if

- $0 \leq i<(p-1) / 2$ and $0 \leq j<8$, or

- $i=(p-1) / 2$ and $0 \leq j<4$,

then

$$
g_{8 i+j}=\langle z\rangle y^{2 i} \cdot\left\{\begin{array}{cl}
e & \text { if } j=0, \\
f^{2} & \text { if } j=1, \\
f & \text { if } j=2, \\
y f^{3} & \text { if } j=3, \\
y f^{2} & \text { if } j=4, \\
y & \text { if } j=5, \\
y f & \text { if } j=6, \\
y^{2} f^{3} & \text { if } j=7 .
\end{array}\right.
$$

All of these are distinct, so Lemma 2.6 tells us we have a hamiltonian cycle.

Subcase 2.3. Assume $|S|=3$ (and $f^{2}$ is nontrivial on $\mathbb{Z}_{p} \times \mathbb{Z}_{p}$ ).

Subsubcase 2.3.1. Assume $x^{f^{2}}=x^{-1}$ and $y^{f^{2}}=y$. Since $f$ obviously commutes with $f^{2}$, it must preserve the eigenspaces of $f^{2}$. So $x^{f} \in\langle x\rangle$ and $y^{f} \in\langle y\rangle$. So $x^{f}=x^{r}$, where $r^{2} \equiv-1(\bmod p)$, and $y^{f}=y^{-1}$. Since $|S|=3$, we know that $S$ does not contain any element of the form $f^{\ell} x^{i} y^{j}$ with $i$ and $j$ both nonzero, so we can clearly assume that $S=\left\{f, f^{\ell_{1}} x, f^{\ell_{2}} y\right\}$. We may also assume $0 \leq \ell_{1}, \ell_{2} \leq 2$ (after replacing generators by their inverses, if necessary).

- If $\ell_{2}=0$, then Lemma 2.27 applies since $\langle y\rangle \triangleleft G$.

- If $\ell_{2}=1$, then Corollary 2.11 applies since $f y \equiv f(\bmod \langle y\rangle)$ and $\langle y\rangle \triangleleft G$.

- If $\ell_{2}=2$, then Corollary 2.11 applies since $\left(f^{2} y\right)^{2}=y^{2}$ and $\left\langle y^{2}\right\rangle=\langle y\rangle \triangleleft G$.

Subsubcase 2.3.2. Assume $x^{f^{2}}=x^{-1}$ and $y^{f^{2}}=y^{-1}$. We may assume $S=$ $\left\{f, f^{\ell_{1}} x, f^{\ell_{2}} y\right\}$. Since $|S|=3$, we must have $x^{f} \in\langle x\rangle$ and $y^{t} \in\langle y\rangle$. Therefore $x^{f}=x^{a}$ and $y^{f}=y^{b}$, where $a$ and $b$ are square roots of -1 in the field $\mathbb{Z}_{p}$.

If either $\ell_{1}$ or $\ell_{2}$ is equal to 0,1 , or 3 , then we may apply Lemma 2.27 or Corollary 2.11, because $\langle x\rangle$ and $\langle y\rangle$ are both normal subgroups of $G$. We are therefore left with $S=$ $\left\{f, f^{2} x, f^{2} y\right\}$. Take $s_{1}=f^{2} x$ and $s_{2}=f^{2} y$. Then $s_{1} s_{2}=x^{-1} y$ is of order $p$ and since $\left|\left\langle f, f^{2} y\right\rangle\right|=4 p$ and $s_{1} s_{2} \notin\left\langle f, f^{2} y\right\rangle$, we see that Lemma 2.18 applies. 


\section{Groups of order $p q r$}

Proposition 6.1. If $|G|=2 p q$, where $p$ and $q$ are prime, then every connected Cayley graph on $G$ has a hamiltonian cycle.

Proof. Let $S$ be a minimal generating set of $G$.

We may assume $p$ and $q$ are distinct, for otherwise $|G|=2 p^{2}$, so Corollary 2.24 applies. Thus, there is no harm in assuming that $p<q$. We may also assume that $p, q \geq 3$, for otherwise $|G|$ is of the form $4 p$, so Corollary 2.17 applies.

Let $Q$ be a Sylow $q$-subgroup of $G$. Because $|G|=2 p q=2 \times$ odd, it is well known that $G$ has a (unique) normal subgroup of order $p q$ [24, Thm. 4.6]. Since $p<q$, Sylow's Theorem (2.33) tells us that $Q$ is normal in this subgroup. Then, being characteristic, $Q$ is normal in $G$.

The quotient group $G / Q$ is of order $2 p$. We may assume it is nonabelian, for otherwise $[G, G]=Q$ is cyclic of prime order, so Theorem 2.2 applies. Therefore $G \cong D_{2 p} \ltimes$ $Q$. Because Aut $Q \cong\left(\mathbb{Z}_{q}\right)^{\times}$is abelian, we know that the commutator subgroup of $D_{2 p}$ centralizes $Q$. Hence $G \cong \mathbb{Z}_{2} \ltimes\left(\mathbb{Z}_{p} \times \mathbb{Z}_{q}\right)$. Then either $G \cong D_{2 p q}$ is dihedral (so Lemma 2.22 applies) or $[G, G]$ has prime order (so Theorem 2.2 applies).

Proposition 6.2 (D. Li [19]). If $|G|=p q r$, where $p$, $q$, and $r$ are distinct primes, then every connected Cayley graph on $G$ has a hamiltonian cycle.

Proof. The case where $|G|=2 p q$ has been discussed in Proposition 6.1, so let us assume $|G|$ is odd.

Also assume $p$ is the smallest of $p, q$ and $r$. Because $|G|$ is square-free, it is well known (and not difficult to prove) that $[G, G]$ must be cyclic of some order dividing $|G| / p[13$, Cor. 9.4.1]. (In particular, $G$ is solvable.) We may assume $|[G, G]|$ is not prime, so we conclude that

$$
[G, G] \cong \mathbb{Z}_{q r}
$$

Thus, $G$ is a semidirect product: up to isomorphism, we have

$$
G=\mathbb{Z}_{p} \ltimes\left(\mathbb{Z}_{q} \times \mathbb{Z}_{r}\right),
$$

where $\mathbb{Z}_{p}$ acts nontrivially on both $\mathbb{Z}_{q}$ and $\mathbb{Z}_{r}$.

Now, let $S$ be a minimal generating set of $G$. Since $\left(\mathbb{Z}_{q} \times \mathbb{Z}_{r}\right) \cap Z(G)=\emptyset$, Lemma 2.27 tells us that we may assume $S \cap\left(\mathbb{Z}_{q} \times \mathbb{Z}_{r}\right)=\emptyset$. Thus, every element of $S$ has order $p$.

Case 1. Assume $|S|=2$. We may write $S=\{s, t\}$. We have $t=x s^{k}$ for some generator $x$ of $\left.\mathbb{Z}_{q} \times \mathbb{Z}_{r}\right)$ and some integer $k$ with $1 \leq k<p$. Then

$$
t s^{-(k-1)} t s^{p-k-1}=x s x s^{-1}
$$

is a generator of $\mathbb{Z}_{q} \times \mathbb{Z}_{r}$ (because $s$, being of odd order, cannot invert either $\mathbb{Z}_{q}$ or $\mathbb{Z}_{r}$ ). So Corollary 2.8 tells us that

$$
\left(t, s^{-(k-1)}, t, s^{p-k-1}\right)^{q r}
$$

is a hamiltonian cycle in $\operatorname{Cay}(G ; S)$.

Case 2. Assume $|S|=3$. We may write $S=\{s, t, u\}$. The minimality of $S$, together with the fact that $S \cap\left(\mathbb{Z}_{q} \times \mathbb{Z}_{r}\right)=\emptyset$, implies $t=s^{i} x$ and $u=s^{j} y$, where $x$ and $y$ are generators of $\mathbb{Z}_{q}$ and $\mathbb{Z}_{r}$, respectively, and $1 \leq i, j<p$. Then $\langle t, u\rangle=G$, which contradicts the fact that the generating set $S$ is minimal. 
Corollary 6.3. If $|G|=3 p q$, where $p$ and $q$ are prime, then every connected Cayley graph on $G$ has a hamiltonian cycle.

Proof. Note that:

- We may assume $p, q \geq 3$, for otherwise $|G|$ is of the form $2 p q$ or $2 p^{2}$ so Proposition 6.1 or Corollary 2.24 applies.

- We may assume $p \neq q$, for otherwise $|G|=3 p^{2}$, so Proposition 4.1 applies.

- We may assume $p, q>3$, for otherwise $|G|$ is of the form $p^{2} q$ with $p \not \equiv 1(\bmod q)$, so Corollary 2.3 applies.

Thus, $|G|$ is the product of three distinct primes, so Proposition 6.2 applies.

Corollary 6.4. If $|G|=5 p q$, where $p$ and $q$ are distinct primes, then every connected Cayley graph on $G$ has a hamiltonian cycle.

Proof. Note that:

- We may assume $p, q \geq 5$, for otherwise $|G|$ is of the form $2 p q$ or $2 p^{2}$ or $3 p q$ or $3 p^{2}$, so Proposition 6.1 or Corollary 2.24 or Corollary 6.3 or Proposition 4.1 applies.

- Then we may assume $p, q \neq 5$, for otherwise $|G|$ is of the form $p^{3}$ or $p^{2} q$ with $p^{2} \not \equiv 1(\bmod q)$, so Remark 1.3 or Corollary 2.3 applies.

Thus, $|G|$ is the product of three distinct primes, so Proposition 6.2 applies.

\section{Groups of order $4 p q$}

We start by considering a special case.

Proposition 7.1. If $G=P_{2} \ltimes \mathbb{Z}_{p q}$ is a semidirect product of a group $P_{2}$ of order 4 and a cyclic group $\mathbb{Z}_{p q}$ of order $p q$, where $p$ and $q$ are distinct odd primes, then every connected Cayley graph on $G$ has a hamiltonian cycle.

Proof. Let $S$ be a minimal generating set of $G$. We may assume $[G, G]=\mathbb{Z}_{p q}$ (otherwise Theorem 2.2 applies).

Case 1. Assume $P_{2} \cong \mathbb{Z}_{4}$, so $G \cong \mathbb{Z}_{4} \ltimes \mathbb{Z}_{p q}$. We can view $G$ as $\mathbb{Z}_{4} \ltimes\left(\mathbb{Z}_{p} \times \mathbb{Z}_{q}\right)$. Let $f$ be a generator of $\mathbb{Z}_{4}$ and let $x$ and $y$ be generators of $\mathbb{Z}_{p}$ and $\mathbb{Z}_{q}$, respectively. There exists $r \in \mathbb{Z}$, such that $x^{f}=x^{r}$ and $y^{f}=y^{r}$. We have $r^{4} \equiv 1(\bmod p q)$, because $|f|=4$.

Note that in view of Lemma 2.27 and the fact that $Z(G) \cap\left(\mathbb{Z}_{p} \times \mathbb{Z}_{q}\right)=\{e\}$, we can assume that no element of the form $x^{j} y^{k}$ is in $S$.

Since $S$ clearly contains at least one element of the form $f^{i} x^{j} y^{k}$, where $i \in\{1,3\}$, we can assume $f \in S$.

Subcase 1.1. Assume the action of $\mathbb{Z}_{4}$ on $\mathbb{Z}_{p q}$ is not faithful. Then $r^{2} \equiv 1(\bmod p q)$. Since $[G, G]=\mathbb{Z}_{p q}$, this implies $r \equiv-1(\bmod p q)$ and thus $f$ inverts every element of $\mathbb{Z}_{p} \times \mathbb{Z}_{q}$. But then $G \cong Q_{4 p q}$ is of quaternion type, so Corollary 2.25 applies.

Subcase 1.2. Assume the action of $\mathbb{Z}_{4}$ on $\mathbb{Z}_{p q}$ is faithful. This means $r^{2} \not \equiv 1(\bmod p q)$, so we may assume $r^{2} \not \equiv 1(\bmod p)$ (by interchanging $p$ and $q$ if necessary). Therefore $r^{2} \equiv-1(\bmod p)$.

Subsubcase 1.2.1. Assume $S$ contains an element $s$ of the form $f x^{j} y^{k}$ or $f^{2} x^{j} y^{k}$, where both $j$ and $k$ are nonzero. Now if $s=f x^{j} y^{k}$, then clearly $f^{3} s=x^{j} y^{k}$ is of order 
$p q$ and thus Corollary 2.8 tells us that $\left(f^{3}, s\right)^{p q}$ is a hamiltonian cycle in $\operatorname{Cay}(G ; S)$. On the other hand, if $s=f^{2} x^{j} y^{k}$, then

$$
f^{-1} s^{-1} f s=f^{-1}\left(x^{j} y^{k}\right)^{-1} f\left(x^{j} y^{k}\right)=\left[f, x^{j} y^{k}\right]
$$

generates $[G, G]=\mathbb{Z}_{p q}$. Thus, it is easy to see from Corollary 2.8 that $\left(f, s, f^{-1}, s^{-1}\right)^{p q}$ is a hamiltonian cycle in $\operatorname{Cay}(G ; S)$.

Subsubcase 1.2.2. Assume all elements of $S-\{f\}$ are of the form $f^{i} x^{j} y^{k}$ where $i \in\{1,2\}$ and precisely one of $j, k$ is nonzero. Clearly, $S=\left\{f, f^{i} x, f^{j} y\right\}$ (perhaps after replacing $x$ and $y$ by their powers). Since $(f x)^{-1} f y=x^{-1} y$, we see that $\langle f x, f y\rangle=G$. Similarly, $\left[f x, f^{2} y\right]=x^{-2}[f, y]$ generates $[G, G]$, and thus $\left\langle f x, f^{2} y\right\rangle=G$. Since $S$ is minimal, there are thus only two possibilities for $S$. First if $s_{1}=f^{2} x, s_{2}=f y \in S$, then $s_{1} f^{-1} s_{1} s_{2}=x^{r-1} y$ is of order $p q$ and thus Corollary 2.8 tells us $\left(s_{1}, f^{-1}, s_{1}, s_{2}\right)^{p q}$ is a hamiltonian cycle in $\operatorname{Cay}(G ; S)$. Finally, if $s_{1}=f^{2} x$ and $s_{2}=f^{2} y$, then $f s_{1} f^{-1} s_{2}=$ $x^{r} y$ is of order $p q$, so Corollary 2.8 tells us $\left(f, s_{1}, f^{-1}, s_{2}\right)^{p q}$ is a hamiltonian cycle in $\operatorname{Cay}(G ; S)$.

Case 2. Assume $P_{2} \cong \mathbb{Z}_{2} \times \mathbb{Z}_{2}$, so $G \cong\left(\mathbb{Z}_{2} \times \mathbb{Z}_{2}\right) \ltimes \mathbb{Z}_{p q}$.

Subcase 2.1. Assume some involution in $\mathbb{Z}_{2} \times \mathbb{Z}_{2}$ centralizes $\mathbb{Z}_{p q}$. (That is, $\mathbb{Z}_{2} \times \mathbb{Z}_{2}$ is not faithful on $\mathbb{Z}_{p q}$.) Then $G \cong \mathbb{Z}_{2} \ltimes \mathbb{Z}_{2 p q} \cong D_{4 p q}$ is dihedral, so Lemma 2.22 applies.

Subcase 2.2. Assume no involution in $\mathbb{Z}_{2} \times \mathbb{Z}_{2}$ centralizes $\mathbb{Z}_{p q}$. (That is, $\mathbb{Z}_{2} \times \mathbb{Z}_{2}$ is faithful on $\mathbb{Z}_{p q}$.) Then $G \cong D_{2 p} \times D_{2 q}$.

If $S$ contains an element $s$ of $\mathbb{Z}_{p q}$, then $\langle s\rangle \triangleleft G$. Also, since $[G, G]=\mathbb{Z}_{p} \times \mathbb{Z}_{q}$, we see that $Z(G) \cap\left(\mathbb{Z}_{p} \times \mathbb{Z}_{q}\right)=\{e\}$. Thus Lemma 2.27 applies.

Henceforth, we assume $S \cap \mathbb{Z}_{p q}=\emptyset$. Also, we consider $G$ to be $D_{2 p} \times D_{2 q}$.

Let us consider the possibility that $f x^{\prime} \in S$, where $f$ is a reflection in $D_{2 p}$, and $x^{\prime}$ is a nontrivial rotation in $D_{2 q}$ (so $x^{\prime}$ generates $\mathbb{Z}_{q}$ ). We may assume that $S-\left\{f x^{\prime}\right\}$ generates $G / \mathbb{Z}_{q}$ (otherwise, Corollary 2.11 applies with $N=\mathbb{Z}_{q}$ ). Furthermore, it is easy to see that the only proper subgroups of $G$ that properly contain $f x^{\prime}$ are $D_{2 p} \times \mathbb{Z}_{q}$ and $\langle f\rangle \times D_{2 q}$. It is therefore not difficult to see that (up to isomorphism) the only possible Cayley graph is:

$$
\operatorname{Cay}\left(D_{2 p} \times D_{2 q} ;\left\{f x^{\prime}, f f^{\prime}, f x\right\}\right) \text { where } f, x \in D_{2 p} \text { and } f^{\prime}, x^{\prime} \in D_{2 q} \text {. }
$$

Note that $\left(f x, f x^{\prime}\right)$ is obviously a hamiltonian cycle in

$$
\operatorname{Cay}\left(\frac{\left\langle f, x, x^{\prime}\right\rangle}{\left\langle x, x^{\prime}\right\rangle} ;\left\{f x, f x^{\prime}\right\}\right) \cong \operatorname{Cay}(\langle f\rangle ;\{f\}),
$$

so, since $(f x)\left(f x^{\prime}\right)=x^{-1} x^{\prime}$ generates $\left\langle x, x^{\prime}\right\rangle$, Corollary 2.8 implies that

$$
\left(\left(f x, f x^{\prime}\right)^{p q} \#\right)
$$

is a hamiltonian path in $\operatorname{Cay}\left(\left\langle f, x, x^{\prime}\right\rangle ;\left\{f x, f x^{\prime}\right\}\right)$. Therefore, all of the vertices of this path are in different right cosets of $\left\langle f^{\prime} x^{\prime}\right\rangle$. So Lemma 2.6 tells us that

$$
\left(\left(f x, f x^{\prime}\right)^{p q} \#, f f^{\prime}\right)^{2}
$$

is a hamiltonian cycle in $\operatorname{Cay}(G ; S)$. 
We may now assume there is no double edge in $\operatorname{Cay}\left(G / \mathbb{Z}_{p} ; S\right)$ or $\operatorname{Cay}\left(G / \mathbb{Z}_{q} ; S\right)$. (That is, if $s \in S$, and $s$ represents an element of order 2 in $G / \mathbb{Z}_{p}$ or $G / \mathbb{Z}_{q}$, then $s$ has order 2 in $G$.) This implies that $S$ consists entirely of elements of order 2 .

If $S$ has four (or more) elements, it is clear from the minimality of $S$ that $S$ has the form $S=\left\{f, f x, f^{\prime}, f^{\prime} x^{\prime}\right\}$ (with $\langle f, x\rangle=D_{2 p} \times\{e\}$ and $\left\langle f^{\prime}, x^{\prime}\right\rangle=\{e\} \times D_{2 q}$. This means that $\operatorname{Cay}(G ; S)$ is isomorphic to the Cartesian product $C_{2 p} \times C_{2 q}$ of two cycles, which obviously has a hamiltonian cycle [4, Cor. on p. 29].

We now assume that $S=\{s, t, u\}$ consists of precisely three elements of order 2 .

Subsubcase 2.2.1. Let assume $S \cap D_{2 p}=\emptyset$ and $S \cap D_{2 q}=\emptyset$. Then $s \equiv t \equiv$ $u\left(\bmod \mathbb{Z}_{p q}\right)$. This is impossible, because $G / \mathbb{Z}_{p q} \cong \mathbb{Z}_{2} \times \mathbb{Z}_{2}$ is not cyclic.

Subsubcase 2.2.2. Assume $s \in S \cap D_{2 p}$ and $t \in S \cap D_{2 q}$. Then $s \in Z\left(G / \mathbb{Z}_{p}\right)$ and $G / \mathbb{Z}_{p} \cong D_{4 q}$. Since $\{t, u\}$ generates $G / \mathbb{Z}_{p}$, and Cay $\left(G / \mathbb{Z}_{p} ;\{t, u\}\right)$ is a $4 q$-cycle, we see that $\operatorname{Cay}\left(G / \mathbb{Z}_{p} ; S\right)$ is isomorphic to $\operatorname{Cay}\left(\mathbb{Z}_{4 q} ;\{1,2 q\}\right)$. So Theorem 2.13 implies that some hamiltonian cycle in $\operatorname{Cay}\left(G / \mathbb{Z}_{p} ; S\right)$ lifts to a hamiltonian cycle in $\operatorname{Cay}(G ; S)$.

Subsubcase 2.2.3. Assume $s \in S \cap D_{2 p}$ and $S \cap D_{2 q}=\emptyset$. Each element of $G=$ $D_{2 p} \times D_{2 q}$ is an ordered pair. Also, since $\{t, u\}$ generates $G / D_{2 p}$, we know that neither $t$ nor $u$ belongs to $D_{2 p}$ (and, by assumption, they do not belong to $\left.D_{2 q}\right)$, so $t \equiv u\left(\bmod \mathbb{Z}_{p q}\right)$. Therefore, we may write

$$
t=\left(f, f^{\prime}\right), s=(f x, e), u=\left(f x^{k}, f^{\prime} x^{\prime}\right),
$$

where $f$ and $f^{\prime}$ are reflections, $x$ and $x^{\prime}$ are nontrivial rotations, and $k \in \mathbb{Z}$.

Subsubsubcase 2.2.3.1. Assume $k \not \equiv 2(\bmod p)$. Since $(s, t, s, u)$ is a hamiltonian cycle in $\operatorname{Cay}\left(G /\left(\mathbb{Z}_{p} \times \mathbb{Z}_{q}\right) ; S\right)$, and

$$
\text { stsu }=\left(f x \cdot f \cdot f x \cdot f x^{k}, e \cdot f^{\prime} \cdot e \cdot f^{\prime} x^{\prime}\right)=\left(x^{k-2}, x^{\prime}\right)
$$

generates $\mathbb{Z}_{p} \times \mathbb{Z}_{q}$ (because $\left.k \not \equiv 2(\bmod p)\right)$, Corollary 2.8 implies that $(s, t, s, u)^{p q}$ is a hamiltonian cycle in $\operatorname{Cay}(G ; S)$.

Subsubsubcase 2.2.3.2. Assume $k \equiv 2(\bmod p)$. We claim that $\operatorname{Cay}(G ; S)$ is a generalized Petersen graph. To see this, begin by letting

$$
x_{2 i}=(u t)^{i} \text { and } x_{2 i+1}=(u t)^{i} u \text { for } 0 \leq i<p q
$$

and

$$
y_{j}=s x_{j} \text { for } 0 \leq j<2 p q .
$$

Then every vertex of $\operatorname{Cay}(G ;\{t, u\})$ is in the union of the two disjoint $2 p q$-cycles

$$
\left(x_{0}, x_{1}, x_{2}, \ldots, x_{2 p q-1}, x_{2 p q}\right) \text { and }\left(y_{0}, y_{1}, y_{2}, \ldots, y_{2 p q-1}, y_{2 p q}\right) \text {. }
$$

Now, write $\left(x^{2}, e\right)=(u t)^{r}$ with $1 \leq r<p q$. Then

$$
\text { sts }=\left(f x^{2}, f^{\prime}\right)=t(u t)^{r} \text { and sus }=\left(f x^{2-k}, f^{\prime} x^{\prime}\right)=\left(f, f^{\prime} x^{\prime}\right)=(u t)^{r} u
$$

so, by induction on $i$, we see that

$$
x_{i} s=s x_{(2 r+1) i}=y_{(2 r+1) i},
$$

which means there is an $s$-edge from $x_{i}$ to $y_{(2 r+1) i}$. Therefore, $\operatorname{Cay}(G ;\{s, t, u\})$ is a generalized Petersen graph, as claimed.

Now, if we let $n=2 p q$, then: 
- The number of vertices of $\operatorname{Cay}(G ; S)$ is $2 n$.

- Since $p q$ is odd, we know that $n=2 p q \neq \equiv 0(\bmod 4)$.

- Since $2 p q$ is even, we know $n=2 p q \not \equiv 5(\bmod 6)$.

Therefore Theorem 2.29 tells us that $\operatorname{Cay}(G ; S)$ has a hamiltonian cycle.

Proposition 7.2. If $|G|=4 p q$, where $p$ and $q$ are prime, then every connected Cayley graph on $G$ has a hamiltonian cycle.

Proof. Let $S$ be a minimal generating set of $G$. We may assume $p$ and $q$ are distinct (for otherwise $|G|=4 p^{2}$, so Proposition 5.3 applies). Furthermore, we may assume $p, q \geq 3$, for otherwise $|G|$ is of the form $8 p$, so Proposition 3.2 applies. We also assume $G \neq A_{5}$ (since Cayley graphs on that group are covered in Lemma 2.32). It then follows easily from Burnside's Theorem on normal $p$-complements that $G$ is solvable.

Let $P_{2}$ be a Sylow 2-subgroup of $G$. Because $G$ is solvable, Proposition 2.37 tells us there are Hall subgroups $H_{p q}$ and $H_{4 q}$ of order $p q$ and $4 q$, respectively.

There is no harm in assuming that $p>q$ (so $p \geq 5$ ). This implies the Sylow $p$-subgroup $\mathbb{Z}_{p}$ is normal in $H_{p q}$. So

$$
\left|G: N_{G}\left(\mathbb{Z}_{p}\right)\right| \leq\left|G: H_{p q}\right|=4<p+1 .
$$

Therefore $\mathbb{Z}_{p} \triangleleft G$. So $G=H_{4 q} \ltimes \mathbb{Z}_{p}$.

Case 1. Assume $H_{4 q}$ has a normal Sylow q-subgroup. We may assume $H_{4 q}$ is not abelian (otherwise Theorem 2.2 applies). This implies the commutator subgroup of $H_{4 q}$ must be a Sylow $q$-subgroup $\mathbb{Z}_{q}$. Because $\operatorname{Aut}\left(\mathbb{Z}_{p}\right)$ is abelian, this implies that $\mathbb{Z}_{q}$ centralizes $\mathbb{Z}_{p}$. So $G=P_{2} \ltimes\left(\mathbb{Z}_{p} \times \mathbb{Z}_{q}\right) \cong P_{2} \ltimes \mathbb{Z}_{p q}$. Therefore Proposition 7.1 applies.

Case 2. Assume the Sylow q-subgroups of $H_{4 q}$ are not normal. Since $H_{4 q}$ is of order $4 q$, Sylow's Theorem (2.33) tells us there is a divisor of 4 that is congruent to 1 modulo $q$. Clearly, we must have $q=3$. Thus, $H_{4 q}$ is a group of order $4 \cdot 3=12$, in which the Sylow 3 -subgroups are not normal. The only such group is $A_{4}$. So $G \cong A_{4} \ltimes \mathbb{Z}_{p}$.

We have $G \cong \mathbb{Z}_{3} \ltimes\left(\left(\mathbb{Z}_{2} \times \mathbb{Z}_{2}\right) \times \mathbb{Z}_{p}\right)$. We may assume $S \cap \mathbb{Z}_{p}=\emptyset$, for otherwise Lemma 2.27 applies.

We let $f$ be a generator of $\mathbb{Z}_{3}, x$ and $y$ be generators of $\mathbb{Z}_{2} \times \mathbb{Z}_{2}$ and $z$ be a generator of $\mathbb{Z}_{p}$ where $x^{f}=x y, y^{f}=x$ and $z^{f}=z^{r}$ for some $r \in \mathbb{Z}$ such that $r^{3} \equiv 1(\bmod p)$.

Subcase 2.1. Assume $\mathbb{Z}_{3}$ acts nontrivially on $\mathbb{Z}_{p}$. Since $S$ must contain an element of $G-\langle x, y, z\rangle$, and all of these elements have order 3 , we can assume $f \in S$.

Subsubcase 2.1.1. Assume $|S|=2$. Let $S=\{f, s\}$.

Subsubsubcase 2.1.1.1. Assume $s \in\left(\mathbb{Z}_{2} \times \mathbb{Z}_{2}\right) \times \mathbb{Z}_{p}$. The generator $s$ gives a double edge in $G / \mathbb{Z}_{p}$ (and $\{f, s\}$ is a minimal generating set of $G / \mathbb{Z}_{p}$ ), so Corollary 2.11 applies.

Subsubsubcase 2.1.1.2. Assume $s \in f\left(\left(\mathbb{Z}_{2} \times \mathbb{Z}_{2}\right) \times \mathbb{Z}_{p}\right)$. Write $s=f x^{i} y^{j} z^{k}$. Since $S$ generates $G$, we cannot have $i=j=0$ or $k=0$, so we can assume $s=f x z$.

We show that $f$ and $s$ satisfy the conditions of Lemma 2.14. We have

$$
[f, s]=f^{-1}(f x z)^{-1} f(f x z)=f^{-1} z^{-1} x^{-1} f^{-1} f^{2} x z=y z^{1-r} .
$$


Since $f$ acts nontrivially on $\mathbb{Z}_{p}$, we know $r \not \equiv 1(\bmod p)$, so $|[f, s]|=2 p$. Also, we have

$$
s=f x z \notin\langle f\rangle\langle[f, s]\rangle .
$$

The other two conditions are clearly satisfied.

Subsubcase 2.1.2. Assume $|S|=3$. We may assume $S=\left\{f, f^{i} x, f^{j} z\right\}$, and $i, j \in\{0,1\}$. Since $S \cap \mathbb{Z}_{p}=\emptyset$, we have $j=1$, for otherwise Lemma 2.27 applies. But then $\{f, f z\}$ gives a double edge in $\operatorname{Cay}\left(G / \mathbb{Z}_{p} ; S\right)$ (and $S-\{f z\}$ is a minimal generating set of $\left.G / \mathbb{Z}_{p}\right)$, so Corollary 2.11 applies.

Subcase 2.2. Assume $\mathbb{Z}_{3}$ centralizes $\mathbb{Z}_{p}$. This means $r=1$, and we have $G \cong A_{4} \times \mathbb{Z}_{p}$. Recalling that $S \cap \mathbb{Z}_{p}=\emptyset$, we know that no element of $S$ has order $p$.

Let $s$ be an element of $S$ whose order is divisible by $p$. Note that $\langle s\rangle$ contains a nontrivial subgroup of $G / \mathbb{Z}_{p} \cong \mathbb{Z}_{3} \ltimes\left(\mathbb{Z}_{2} \times \mathbb{Z}_{2}\right)$. Either this subgroup is maximal (of order 3 ) or we have $\langle s, t\rangle=G$ for any $t \in S$ with 3||$t \mid$. Therefore $|S|=2$, so we may write $S=\{s, t\}$.

We may assume $s \notin\left(\mathbb{Z}_{2} \times \mathbb{Z}_{2}\right) \times \mathbb{Z}_{p}$, for otherwise $\left\{s, s^{-1}\right\}$ gives a double edge in $\operatorname{Cay}\left(G / \mathbb{Z}_{p} ; S\right)$, so Corollary 2.11 applies. Therefore, we may assume $s=f z$.

We show that $f z$ and $t$ satisfy the conditions of Lemma 2.14. Since $\langle f z, t\rangle=G$, we may assume $t=f^{\ell} x z^{k}$ for some $\ell, k \in \mathbb{Z}$. Then

$$
[f z, t]=\left[f z, f^{\ell} x z^{k}\right]=y .
$$

Since all nonidentity elements of $\langle f z\rangle$ are in $\langle f\rangle\langle z\rangle$, we see that $t \notin\langle f z\rangle\langle[s, t]\rangle$, and the remaining conditions are also clearly satisfied.

\section{Groups of order $2 p^{3}$}

Proposition 8.1. If $|G|=2 p^{3}$, where $p$ is prime, then every connected Cayley graph on $G$ has a hamiltonian cycle.

Proof. Let $S$ be a minimal generating set of $G$.

We may assume $p \geq 3$. (Otherwise, $|G|=2^{4}$ is a prime power, so Remark 1.3 applies.) Let $P$ be a Sylow $p$-subgroup of $G$, so $G=\mathbb{Z}_{2} \ltimes P$, and let $f$ be a generator of $\mathbb{Z}_{2}$.

We may assume $S \not \subset f P$ (for otherwise Corollary 2.4 applies). Thus, there exists $s \in S \cap P$.

Case 1. Assume $\langle s\rangle \triangleleft G$. Note that if $|s|=p$, then Lemma 2.27 applies. Also, if $|s|=p^{3}$, then $\langle s\rangle=P \supset[G, G]$, so $[G, G]$ is cyclic of $p$-power order, so Theorem 2.2 applies. Thus, we may assume $|s|=p^{2}$.

Also, we may assume $\langle s\rangle \cap Z(G)$ is nontrivial (else Lemma 2.27 applies), so it is clear that $f$ does not invert $\langle s\rangle$. Since $|f|=2$, we conclude that $f$ centralizes $s$. Since we may assume that $|[G, G]| \neq p$ (else Theorem 2.2 applies), this implies that we may assume $P$ is nonabelian.

Now, for any $x \in P$, we have $\langle[x, s]\rangle \subset\langle s\rangle$ (because $\langle s\rangle \triangleleft G$ ), so $f$ centralizes $[s, x]$. Therefore

$$
\left[x^{f}, s\right]=\left[x^{f}, s^{f}\right]=[x, s]^{f}=[x, s]
$$


so $f$ centralizes $x$, modulo $C_{P}(s)=\langle s\rangle$. Thus, $f$ centralizes both $P /\langle s\rangle$ and $\langle s\rangle$. Since $|f|=2$ is relatively prime to $|P|$, this implies that $f$ centralizes $P$ (see [11, Thm. 5.3.2]). Therefore $G=\mathbb{Z}_{2} \times P$, so $[G, G]=[P, P]$ is cyclic of order $p$, so Theorem 2.2 applies.

Case 2. Assume $\langle s\rangle \not G$. There is an element $a$ of $S$ with $|a|$ even.

Subcase 2.1. Assume $G=\mathbb{Z}_{2} \times P$. Then $[G, G]$ is cyclic of order 1 or $p$, so Theorem 2.2 applies.

Subcase 2.2. Assume $P$ is abelian (but $G$ is nonabelian). We may assume $G$ is not of dihedral type (else Proposition 2.23 applies). So $|[G, G]| \leq p^{2}$. We may also assume $[G, G]$ is not cyclic (for otherwise Theorem 2.2 applies). Therefore $P=\left(\mathbb{Z}_{p}\right)^{3}$, and

$$
G=\left(\mathbb{Z}_{2} \ltimes\left(\mathbb{Z}_{p} \times \mathbb{Z}_{p}\right)\right) \times \mathbb{Z}_{p}=\left\langle\begin{array}{l|l}
f, x, y, z & \begin{array}{c}
f^{2}=x^{p}=y^{p}=z^{p}=e, \\
x^{f}=x^{-1}, y^{f}=y^{-1}, z^{f}=z, \\
\langle x, y, z\rangle \text { is abelian }
\end{array}
\end{array}\right\rangle
$$

is the direct product of a group of dihedral type with a cyclic group of order $p$. Also note that, because $a^{2}$ is in the elementary abelian group $P$, we have $\left|a^{2}\right| \in\{1, p\}$.

Since any two elements of order 2 always generate a dihedral group, it is easy to see that $G / Z(G)$ has no 2-element generating set. Therefore $|S| \geq 3$.

Subsubcase 2.2.1. Assume $a^{2} \neq e$. We know $a^{2}$ is in $Z(G)$ (because it is centralized by both $a$ and the abelian group $P$ ), so we conclude that $\left\langle a^{2}\right\rangle=Z(G)$ is normal in $G$. Also, since $\langle a\rangle$ has index $p^{2}$ in $G$, we know that $|S| \leq 3$. Then, since $G / Z(G)$ has no 2-element generating set, we conclude that $S$ is a minimal generating set of $G /\left\langle a^{2}\right\rangle$. Thus, Corollary 2.11 (with $N=\left\langle a^{2}\right\rangle$ and $s=a=t^{-1}$ ) provides a hamiltonian cycle in $\operatorname{Cay}(G ; S)$.

Subsubcase 2.2.2. Assume $a^{2}=e$. We may assume $f \in S$. Since $\langle s\rangle \not G$, we must have $|\langle f, s\rangle|>2 p$. Therefore, the minimality of $S$ implies $|S| \leq 3$. Since we already have the opposite inequality, we conclude that $|S|=3$; write $S=\{f, s, t\}$.

- Suppose $t \notin P$. Then we may assume $t^{2}=e$ (otherwise Subsubcase 2.2.1 applies), so $f t$ is inverted by $f$, so it generates a normal subgroup of $G$. Since $f \equiv$ $t(\bmod \langle f t\rangle)$, the multigraph Cay $(G /\langle f t\rangle ; S)$ has double edges, and it is clear that all of its hamiltonian cycles use at least one of these double edges (since $\langle f t, s\rangle \neq G$ ). Therefore Corollary 2.9 applies.

- Suppose $t \in P$ (and $\langle t\rangle \Varangle G$, so Case 1 does not apply). We may assume $s=x z$ and $t=y z^{k}$ for some $k \not \equiv 0(\bmod p)$ (because $\langle s\rangle$ and $\langle t\rangle$ are not normal). We have $\left(t^{-1} f\right)^{2}=z^{-2 k} \in Z(G)$. Since $\langle s, t\rangle \cap Z(G)=\{e\}$, it is therefore clear that $\langle s, t\rangle \cap\left\langle t^{-1} f\right\rangle=\{e\}$, so all of the elements of $\langle s, t\rangle$ are in different right cosets of $\left\langle t^{-1} f\right\rangle$. Since $\left(s^{p-1}, t\right)^{p} \#$ is a hamiltonian path in Cay $(\langle s, t\rangle ;\{s, t\})$, this implies that all of the vertices in this path are in different right cosets of $\left\langle t^{-1} f\right\rangle$.

Then, since $\left|t^{-1} f\right|=2 p=|G| /|\langle s, t\rangle|$, Lemma 2.6 tells us that $\left(\left(s^{p-1}, t\right)^{p} \#, f\right)^{2 p}$ is a hamiltonian cycle in $\operatorname{Cay}(G ; S)$.

Subcase 2.3. Assume $P$ is nonabelian of exponent $p^{2}$. We have

$$
\left.P=\langle x, y| x^{p^{2}}=y^{p}=e,[x, y]=x^{p} \text { is central }\right\rangle .
$$


Since $\langle[P, P], y\rangle$ is the unique elementary abelian subgroup of order $p^{2}$ in $G$, it must be normalized by $f$. Thus, $\langle y\rangle$ must be in an eigenspace of the action of $f$ on $P /[P, P]$, so we may assume $y^{f} \in\left\{y^{ \pm 1}\right\}$. Also, by choosing $\langle x\rangle$ to also be in an eigenspace, we may assume $x^{f} \in\langle x\rangle$.

Since Aut $(\langle x\rangle)$ is abelian, and $y$ acts nontrivially on $\langle x\rangle$, we know that $y$ is not in the commutator subgroup of $\langle f, y\rangle$. So $f$ cannot invert $y$. Therefore $f$ centralizes $y$. Thus, $[G, G] \subset\langle x\rangle$, so $[G, G]$ is cyclic of prime-power order, so Theorem 2.2 applies.

Subcase 2.4. Assume $P$ is nonabelian of exponent $p$. We have

$$
\left.P=\langle x, y, z| x^{p}=y^{p}=z^{p}=e, z=[x, y] \text { is central }\right\rangle .
$$

We may assume $S \cap\langle z\rangle=\emptyset$, for otherwise Lemma 2.27 applies.

Subsubcase 2.4.1. Assume $|S|=2$. We have $S=\{a, s\}$.

Subsubsubcase 2.4.1.1. Assume $a^{2} \neq e$. We may assume $a^{2} \notin[P, P]$ (otherwise Corollary 2.11 applies), so there is no harm in assuming $a^{2}=y^{2}$ (and $a$ obviously centralizes $\left\langle a^{2}\right\rangle=\langle y\rangle$ ).

Note that, since $a \in f P$, the elements $a$ and $f$ have the same action on $P /[P, P]$, and they have the same action on $Z(P)$.

Since $f$ acts as an automorphism of order 2 on $P /[P, P]$, and does not centralize $P$, it must act nontrivially on $P /[P, P]$, so -1 must be an eigenvalue of this action. Thus, we may assume $x^{f} \in x^{-1}[P, P]$. Then, since $\left[x^{-1}, y\right]=[x, y]^{-1}$ (because $[x, y] \in Z(P)$ ), we have

$$
z^{f}=[x, y]^{f}=\left[x^{f}, y^{f}\right]=\left[x^{-1}, y\right]=[x, y]^{-1}=z^{-1},
$$

so $f$ inverts $\langle z\rangle$. Since $x^{f} \in x^{-1}\langle z\rangle$ (and $\langle x, z\rangle$ is abelian), this implies that

$$
f \text { inverts }\langle x, z\rangle \text {. }
$$

(So $x^{f}=x^{-1}, y^{f}=y$, and $z^{f}=z^{-1}$.) Therefore, replacing $a$ by an appropriate conjugate, we may assume $a=f y$.

- If $s^{a} \in s^{-1}[P, P]$, then we may assume $s=x$. Also, since $[x, y]=z \in Z(P)$, we see that

$$
x x^{a}=x x^{f y}=x\left(x^{-1}\right)^{y}=\left[x^{-1}, y\right]=[x, y]^{-1}=z^{-1}
$$

generates $\langle z\rangle$. Hence, the path

$$
\left(x^{-(p-1)}, a^{-1}, x^{-(p-1)}, a\right)^{p} \#
$$

visits all of the elements of $\langle x, z\rangle \cup a^{-1}\langle x, z\rangle$, so all of the vertices in this path are in different right cosets of $\langle y\rangle$. Then, since $a^{-2}=y^{-1}$ generates $\langle y\rangle$, Lemma 2.6 tells us that

$$
\left(\left(x^{-(p-1)}, a^{-1}, x^{-(p-1)}, a\right)^{p} \#, a^{-1}\right)^{p}
$$

is a hamiltonian cycle in $\operatorname{Cay}(G ; S)$. 
- If $s^{a} \notin s^{-1}[P, P]$, then we may write $s=x y^{\ell}$ with $\ell \not \equiv 0(\bmod p)$, and we may assume $\ell \not \equiv 1(\bmod p)$, by replacing $a$ and $y$ with their inverses if necessary.

Since $s \equiv x(\bmod \langle y, z\rangle)$, and $a$ inverts $x(\bmod \langle y, z\rangle)$, it is clear that $\left(s^{p-1}, a\right)^{2}$ is a hamiltonian cycle in $\operatorname{Cay}(G /\langle y, z\rangle ; S)$. Then, since the product $\left(s^{p-1} a\right)^{2}=$ $y^{2-2 \ell} z^{\ell-1}$ generates $\langle y, z\rangle /\langle y\rangle$, Lemma 2.12 tells us that $\left(s^{p-1}, a\right)^{2 p}$ is a hamiltonian cycle in the quotient $\langle y\rangle \backslash \operatorname{Cay}(G ; S)$. But

$$
a^{-1}=a^{-2} a=y^{-2} a \in\langle y\rangle a,
$$

so the final edge of this hamiltonian cycle is a multiple edge in the quotient. Thus, Corollary 2.9 provides a hamiltonian cycle in $\operatorname{Cay}(G ; S)$.

Subsubsubcase 2.4.1.2. Assume $a^{2}=e$. We may assume $a=f$. Since $\left\langle s, s^{f},[P, P]\right\rangle \triangleleft G$, we have

$$
G=\langle f, s\rangle=\langle f\rangle\left\langle s, s^{f},[P, P]\right\rangle,
$$

so $\left\{s, s^{f}\right\}$ must generate $P /[P, P]$. So $s^{f} \notin\langle s\rangle[P, P]$, which implies that the action of $f$ on $P /[P, P]$ has two distinct eigenvalues (both 1 and -1 ), and that $s$ is not in either of these eigenspaces. Thus, we may assume $s=x y$ with $x^{f}=x^{-1}$ and $y^{f}=y$. Note that, from the calculation of (8.2), we also know $z^{f}=z^{-1}$.

We claim that

$$
\left(\left(s^{p-1}, f\right)^{2 p-2},\left(s^{-(p-1)}, f\right)^{2}\right)^{p}
$$

is a hamiltonian cycle in $\operatorname{Cay}(G ; S)$. This walk is obviously of the correct length, and is closed (because $P$ has exponent $p$ ), so we need only show that it visits all of the elements of $G$.

We have

$$
\begin{aligned}
\left(s^{p-1} f\right)^{2 p-2}\left(s^{-(p-1)} f\right)^{2} & =\left(s^{-1} f\right)^{-2}(s f)^{2}=y^{4} \\
\left(s^{p-1} f\right)^{2} & =\left(s^{-1} f\right)^{2}=y^{-2} z, \\
s & =x y
\end{aligned}
$$

so the walk visits all vertices of the form $\left(y^{4}\right)^{i}\left(y^{-2} z\right)^{j}(x y)^{k}$. That is, it visits all of the vertices in $P$.

Also, note that the first vertex of $f P$ visited is $s^{p-1} f$, and we have

$$
\left(s^{p-1} f\right)^{2 p-3}\left(s^{-(p-1)} f\right)^{2}\left(s^{p-1} f\right)=\left(s^{-1} f\right)^{-3}(s f)^{2}\left(s^{-1} f\right)=y^{4} z^{-4},
$$

so the walk visits all vertices of the form

$$
\left(s^{p-1} f\right) \cdot\left(y^{4} z^{-4}\right)^{i}\left(y^{-2} z\right)^{j}(x y)^{k}
$$

with $0 \leq j \leq p-2$. In addition, since

$$
\begin{aligned}
\left(s^{p-1} f\right)^{2 p-3}\left(s^{-(p-10} f\right) & =\left(s^{-1} f\right)^{-3}(s f) \\
& =y^{4} x^{-2} z^{-4} \\
& =\left(y^{4} z^{-4}\right)\left(y^{-2} z\right)^{-1}(x y)^{-2} \\
& \in\left\langle y^{4} z^{-4}\right\rangle\left(y^{-2} z\right)^{p-1}\langle x y\rangle,
\end{aligned}
$$


it also visits the vertices of the form (8.3) with $j=p-1$. Thus, the walk visits all of the vertices in $f P$.

So the walk visits all of the vertices in $P \cup f P=G$, as claimed.

Subsubcase 2.4.2. Assume $|S|=3$. Because $S$ is minimal, we must have $a^{2} \in$ $[P, P]$, so $\left\langle a^{2}\right\rangle \triangleleft G$. So we may assume $a^{2}=e$ (otherwise Corollary 2.11 applies). Thus, we may assume $a=f$. We may also assume $s=x$, so we write $S=\{f, x, t\}$.

Subsubsubcase 2.4.2.1. Assume $t \in P$. We may assume $S=\{f, x, y\}$.

- Suppose $x^{f} \in\left\{x^{ \pm 1}\right\}$. From Remark 1.3, we know there is a hamiltonian cycle $\left(s_{i}\right)_{i=1}^{p^{3}}$ in $\operatorname{Cay}(P ;\{x, y\})$. We may assume $s_{p^{3}}=x^{-1}$. There is also a hamiltonian cycle $\left(t_{i}\right)_{i=1}^{p^{3}}$ in Cay $(P ;\{x, y\})$, such that $t_{p^{3}}=x^{f}$. Then

$$
\left(\left(s_{i}\right)_{i=1}^{p^{3}} \#, f,\left(t_{i}\right)_{i=1}^{p^{3}} \#, f\right)
$$

is a hamiltonian cycle in $\operatorname{Cay}(G ; S)$, because it traverses all the vertices in $P$, then all of the vertices in $f P$, and the final vertex is

$$
\left(s_{1} s_{2} \cdots s_{p^{3}}\right)\left(s_{p^{3}}^{-1} f\right)\left(t_{1} t_{2} \cdots t_{p^{3}}\right)\left(t_{p^{3}}^{-1} f\right)=(e)\left(x^{-1} f\right)(e)\left(x^{f} f\right)=e .
$$

- Suppose $x^{f} \notin\left\{x^{ \pm 1}\right\}$ and $y^{f} \notin\left\{y^{ \pm 1}\right\}$. Because $S$ is minimal, we know that $x^{f} \in$ $\langle x,[P, P]\rangle$ and $y^{f} \in\langle y,[P, P]\rangle$. Since 1 cannot be the only eigenvalue of $f$ on $P /[P, P]$, this means we may assume $x^{f} \in x^{-1}[P, P]$ (by interchanging $x$ and $y$ if necessary).

We claim that $f$ inverts $P /[P, P]$. If not, then $f$ centralizes $y(\bmod [P, P])$, so, from the calculation of (8.2), we see that $f$ inverts $[P, P]$. This implies that $f$ does not centralize any element of $\langle x, z\rangle$, so it must invert all of these elements. This contradicts the assumption that $x^{f} \notin\left\{x^{ \pm 1}\right\}$.

The above claim implies that $\left\langle y x^{-1}, z\right\rangle \triangleleft G$, and that $\left(x^{p-1}, f\right)^{2}$ is a hamiltonian cycle in $\operatorname{Cay}\left(G /\left\langle y x^{-1}, z\right\rangle ;\{f, x\}\right)$. Also note that $\left(x^{p-1} f\right)^{2} \in[P, P]$, and we have

$$
x y x^{p-3} f x^{p-1} f=\left(x y x^{-2}\right)\left(x^{p-1} f\right)^{2}=\left(\left(y x^{-1}\right)\left[y x^{-1}, x^{-1}\right]\right)\left(x^{p-1} f\right)^{2} .
$$

Thus, either $x y x^{p-3} f x^{p-1} f$ or $\left(x^{p-1} f\right)^{2}$ generates $\left\langle y x^{-1},[P, P]\right\rangle /\left\langle y x^{-1}\right\rangle$, depending on whether $\left(x^{p-1} f\right)^{2}$ is trivial or not. Hence, Lemma 2.12 tells us that either

$$
\left(x, y, x^{p-3}, f, x^{p-1}, f\right)^{p} \text { or }\left(x^{p-1}, f\right)^{2 p}
$$

is a hamiltonian cycle in $\left\langle y x^{-1}\right\rangle \backslash \operatorname{Cay}(G ; S)$. Then, since $y \in\left\langle y x^{-1}\right\rangle x$, the first edge of the hamiltonian cycle is doubled in the quotient multigraph, so Corollary 2.9 provides a hamiltonian cycle in $\operatorname{Cay}(G ; S)$.

Subsubsubcase 2 .4.2.2. Assume $t \notin P$. This is very similar to the preceding argument. Write $t=f y$, so $S=\{f, f y, x\}$.

From the argument at the start of Subsubcase 2.4.2, we see that we may assume $(f y)^{2}=$ $e$. Therefore $f$ inverts $y$, so $\langle y,[P, P]\rangle \triangleleft G$. 
Also, since the minimality of $S$ tells us $\langle f, x\rangle \neq G$, we know $x^{f} \in\langle x,[P, P]\rangle$, so there exists $\epsilon \in\{ \pm 1\}$ such that $x^{f} \in x^{\epsilon}[P, P]$. Then it is easy to see that $\left(f, x^{-(p-1)}, f, x^{\epsilon(p-1)}\right)$ is a hamiltonian cycle in $\operatorname{Cay}(G /\langle y, z\rangle ;\{f, x\})$. For

$$
z_{1}=(f)\left(x^{-(p-1)}\right)(f)\left(x^{\epsilon(p-1)}\right)=f x f x^{-\epsilon} \in[P, P]
$$

we have

$$
f\left(x^{-(p-1)}\right)(f y)\left(x^{\epsilon(p-1)}\right)=f x(f y) x^{-\epsilon}=f x f x^{-\epsilon} y\left[y, x^{-\epsilon}\right]=z_{1} y\left[y, x^{-\epsilon}\right] .
$$

Thus, either $(f)\left(x^{-(p-1)}\right)(f y)\left(x^{\epsilon(p-1)}\right)$ or $(f)\left(x^{-(p-1)}\right)(f)\left(x^{\epsilon(p-1)}\right)$ generates

$$
\langle y,[P, P]\rangle /\langle y\rangle,
$$

depending on whether $z_{1}$ is trivial or not. Hence, either

$$
\left(f, x^{-(p-1)}, f y, x^{\epsilon(p-1)}\right)^{p} \text { or }\left(f, x^{-(p-1)}, f, x^{\epsilon(p-1)}\right)^{p}
$$

is a hamiltonian cycle in $\langle y\rangle \backslash \operatorname{Cay}(G ;\{f, f y, x\})$. Then, since $f y \in\langle y\rangle f$ (recall that $f$ inverts $y$ ), the first edge of the hamiltonian cycle is doubled in the quotient multigraph, so Corollary 2.9 provides a hamiltonian cycle in $\operatorname{Cay}(G ; S)$.

\section{Groups of order $18 p$}

Proposition 9.1. If $|G|=18 p$, where $p$ is prime, then every connected Cayley graph on $G$ has a hamiltonian cycle.

Proof. Let $S$ be a minimal generating set of $G$. We may assume $p \geq 5$. (Otherwise either $|G|=36=4 \cdot 3^{2}$, so Proposition 5.3 applies, or $|G|=54=2 \cdot 3^{3}$, so Proposition 8.1 applies.)

Note that $G$ is solvable (for example, this follows from the fact that $|G|=2 \times$ odd, but can be proved quite easily), so Proposition 2.37 tells us $G$ has a Hall subgroup $H_{18}$ of order 18, and also has a Hall subgroup $H_{9 p}$ of order $9 p$. Now $H_{9 p}$, being of index two, is normal in $G$. Also, from Sylow's Theorem (2.33), we see that the Sylow $p$-subgroup $\mathbb{Z}_{p}$ is normal (hence, characteristic) in $H_{9 p}$. So $\mathbb{Z}_{p}$ is normal in $G$. Therefore $G=H_{18} \ltimes \mathbb{Z}_{p}$.

We may assume $H_{18}$ is nonabelian (otherwise Theorem 2.2 applies), so $H_{18}$ is either $D_{18}, \mathbb{Z}_{2} \ltimes\left(\mathbb{Z}_{3} \times \mathbb{Z}_{3}\right)$ (dihedral type), or $D_{6} \times Z_{3}$.

Case 1. Assume $H_{18} \cong D_{18}$. Then either $[G, G]=\mathbb{Z}_{9}$ (so Theorem 2.2 applies) or $G \cong$ $D_{18 p}$ (so Lemma 2.22 applies).

Case 2. Assume $H_{18}=\mathbb{Z}_{2} \ltimes\left(\mathbb{Z}_{3} \times \mathbb{Z}_{3}\right)$ is of dihedral type. We may assume $G$ is not of dihedral type (otherwise Proposition 2.23 applies), so $G=H_{18} \times \mathbb{Z}_{p}$. Let $s$ be an element of $S$ whose order is divisible by $p$.

Subcase 2.1. Assume $|s|=p$. Then $s \in \mathbb{Z}_{p}=Z(G)$, so Lemma 2.27 applies.

Subcase 2.2. Assume $|s|=2 p$. Since $|G|$ is the product of only four primes, and $|s|$ is divisible by two of them, it is clear that $|S| \leq 3$. On the other hand, it is clear that $G / \mathbb{Z}_{p} \cong H_{18}$ has no 2 -element generating set (because two elements of order 2 always 
generate a dihedral group). Therefore $S$ is a minimal generating set of $G / \mathbb{Z}_{p}$. Then, since $s$ and $s^{-1}$ give a double edge in $\operatorname{Cay}\left(G / \mathbb{Z}_{p} ; S\right)$, Corollary 2.11 applies.

Subcase 2.3. Assume $|s|=3 p$. Let $f$ be a generator of $\mathbb{Z}_{2}, x$ and $y$ be generators of $\mathbb{Z}_{3} \times \mathbb{Z}_{3}$ and $z$ be a generator of $\mathbb{Z}_{p}$. We may assume $S$ does not contain any elements of order $p$ or $2 p$ (otherwise, a preceding case applies). We may also assume $S$ does not contain any elements of order 3 (else Lemma 2.27 applies with $N=\mathbb{Z}_{p}$ ). Thus, each element of $S$ has order 2 or $3 p$, so there are only two cases to consider:

Subsubcase 2.3.1. Assume $S=\{f, f x, y z\}$. Since $e, y z$ and $(y z)^{2}$ are in different right cosets of $\left\langle(y z)^{2} f\right\rangle=\langle f y, z\rangle$, Lemma 2.6 tells us that

$$
\left((y z)^{2}, f\right)^{2 p} \#
$$

is a hamiltonian path in the subgraph induced by $\langle f y, z\rangle$. Therefore, all of the vertices of this path are in different right cosets of $\langle x\rangle$. So Lemma 2.6 tells us that

$$
\left(\left((y z)^{2}, f\right)^{2 p} \#, f x\right)^{3}
$$

is a hamiltonian cycle in $\operatorname{Cay}(G ; S)$.

Subsubcase 2.3.2. Assume $S=\left\{f, y z, x z^{k}\right\}$, with $k \neq 0(\bmod p)$. We may assume thet $k \not \equiv 3(\bmod p)$ (by replacing $x z^{k}$ with its inverse, if necessary). Since $G /\left\langle x y^{-1}, z\right\rangle \cong D_{6}$, it is easy to see that

$$
\left(x z^{k}, y z, f,(y z)^{2}, f\right)
$$

is a hamiltonian cycle in $\operatorname{Cay}\left(G /\left\langle x y^{-1}, z\right\rangle ; S\right)$. Then, since

$$
\left(x z^{k}\right)(y z)(f)(y z)^{2}(f)=x y^{-1} z^{k+3}
$$

generates $\left\langle x y^{-1}, z\right\rangle$, Corollary 2.8 tells us that

$$
\left(x z^{k}, y z, f,(y z)^{2}, f\right)^{3 p}
$$

is a hamiltonian cycle in $\operatorname{Cay}(G ; S)$.

Case 3. Assume $H_{18}=D_{6} \times \mathbb{Z}_{3}$. We let $f$ and $x$ generate $D_{6}$, where $f^{2}=x^{3}=e$ and $x^{f}=x^{-1}$. We let $y$ generate $\mathbb{Z}_{3}$ and we let $z$ generate $\mathbb{Z}_{p}$. Note that $y^{f}=y^{x}=y$, and that $z^{x}=z$ (since $x$ is in the commutator subgroup of $H$ ). We may assume $H_{18}$ does not centralize $\mathbb{Z}_{p}$ (otherwise Theorem 2.2 applies). This implies that $C_{G}\left(\mathbb{Z}_{p}\right) /[G, G]$ is a proper subgroup of $G /[G, G] \cong \mathbb{Z}_{2} \times \mathbb{Z}_{3}$, so there are three possibilities for $G$ :

- $G=D_{6 p} \times \mathbb{Z}_{3}$, or

- $G=D_{6} \times\left(\mathbb{Z}_{3} \ltimes \mathbb{Z}_{p}\right)$, or

- $\left.G=\left(D_{6} \times \mathbb{Z}_{3}\right) \ltimes \mathbb{Z}_{p}\right)$, where $D_{6}$ and $\mathbb{Z}_{3}$ both act nontrivially on $\mathbb{Z}_{p}$.

In each case, since $Z(G) \cap\langle x, z\rangle=\{e\}$, we may assume

$$
S \cap\langle x, z\rangle=\emptyset
$$


(else Lemma 2.27 applies).

Subcase 3.1. Assume $G=D_{6 p} \times \mathbb{Z}_{3}$. This implies $z^{f}=z^{-1}$ and $z^{y}=z$. There exists an element of the form $f x^{i} y^{j} z^{k}$ in $S$.

Subsubcase 3.1.1. Assume there exists an element of the form $f x^{i} y z^{k}$ in $S$. Conjugating by powers of $x$ and $z$, we can assume $f y \in S$. Now $(f y)^{2}=y^{2}$ and thus, since $\langle y\rangle \triangleleft G$, either Corollary 2.11 applies or $S-\{f y\}$ generates $G /\langle y\rangle$. Assume the latter. This clearly implies that another element of the form $f x^{i} y^{j} z^{k}$ is in $S$ and that $|S| \geq 3$. Since the index of $\langle f y\rangle$ is $3 p$, which has only two prime factors, we conclude that $|S|=3$. Thus, the minimality of $S$ implies that precisely one of $i$ and $k$ must be zero.

Since the minimality of $S$ implies no element of the form $x y^{j^{\prime}} z$ is in $S$, and since neither $\left\{f x y^{j^{\prime}}, y^{j^{\prime \prime}} z\right\}$ nor $\left\{f y^{j^{\prime}} z, x y^{j^{\prime \prime}}\right\}$ generates $G /\langle y\rangle$, it follows that $S=\left\{f y, f x y^{j}, f y^{j^{\prime}} z\right\}$. But since $S$ is minimal, we must have $j=j^{\prime}=0$. Thus $S=\{f y, f x, f z\}$. Now

- $(f z, f x)^{3 p} \#$ is a hamiltonian path in Cay $(\langle f, x, z\rangle ;\{f z, f x\})$, so all the vertices in this path are obviously in different right cosets of $\left\langle x^{-1} y\right\rangle$, and

- $((f z)(f x))^{3 p}(f x)^{-1}(f y)=x^{-1} y$ obviously generates $\left\langle x^{-1} y\right\rangle$,

so Lemma 2.6 implies that

$$
\left((f z, f x)^{3 p} \#, f y\right)^{3}
$$

is a hamiltonian cycle in $\operatorname{Cay}(G ; S)$.

Subsubcase 3.1.2. Assume that $S$ does not contain any element of the form $f x^{i} y^{j} z^{k}$ with $j \not \equiv 0(\bmod 3)$. Then we can assume $f \in S$. There must be an element of the form $x^{i} y z^{k}$ in $S$. Note that we can assume that at least one of $i$ and $k$ is nonzero for otherwise Lemma 2.27 applies.

Subsubsubcase 3.1.2.1. Assume $i$ and $k$ are both nonzero. Then we can assume $S=\{f, x y z\}$. Since $(x y z)^{3 p-1}$ is a hamiltonian path in Cay $(\langle x y, z\rangle ;\{x y z\})$, it is clear that all of the vertices in this path are in different right cosets of $\langle f x z, y\rangle=\left\langle(x y z)^{3 p-1} f\right\rangle$. So Corollary 2.8 tells us that $\left((x y z)^{3 p-1}, f\right)^{6}$ is a hamiltonian cycle in $\operatorname{Cay}(G ; S)$.

Subsubsubcase 3.1.2.2. Assume $i \neq 0$ and $k=0$. We can assume $x y \in S$. Then, since $S \cap\langle x, z\rangle=\emptyset$, the only candidates for the third element of $S$ are $y z$ and $f x^{i} z$.

- Suppose $y z \in S$. Note that every element of the abelian group $\langle x, y, z\rangle$ can be written uniquely in the form $(y z)^{i}(x y)^{-j}$, where $0 \leq i<3 p$ and $0 \leq j<3$, so it is easy to see that

$$
\left((y z)^{3 p-1},(x y)^{-1},(y z)^{-(3 p-1)},(x y)^{-1},(y z)^{3 p-1}\right)
$$

is a hamiltonian path in $\operatorname{Cay}(\langle x, y, z\rangle ;\{x y, y z\})$. Thus, letting

$$
g=(y z)^{3 p-1}(x y)^{-1}(y z)^{-(3 p-1)}(x y)^{-1}(y z)^{3 p-1} f=x z^{-1} f,
$$

it is clear that all of the vertices of this path are in different right cosets of $\langle g\rangle$ (since $|g|=2$ ). Therefore Lemma 2.6 tells us that

$$
\left((y z)^{3 p-1},(x y)^{-1},(y z)^{-(3 p-1)},(x y)^{-1},(y z)^{3 p-1}, f\right)^{2}
$$

is a hamiltonian cycle in $\operatorname{Cay}(G ; S)$. 
- Suppose $f x^{i} z \in S$. We may assume that $i \not \equiv 0(\bmod 3)$, for otherwise $f \equiv$ $f x^{i} z(\bmod \langle z\rangle)$, so Corollary 2.11 applies with $N=\langle z\rangle$. Then we may assume $i=1$ (by replacing $x$ and $x y$ by their inverses, if necessary). So $S=\{f, f x z, x y\}$. Now, $(x y)^{2} f(x y)^{-2} f x z=x^{2} z$, which generates the normal cyclic subgroup $\langle x, z\rangle=$ $\langle x z\rangle$. Then, since

$$
1, x y, x^{2} y^{2}, f x y^{2}, f y, f x^{2}
$$

lie in different cosets of the subgroup $\langle x z\rangle$, Corollary 2.8 implies that $\left((x y)^{2}, f\right.$, $\left.(x y)^{-2}, f x z\right)^{3 p}$ is a hamiltonian cycle in $\operatorname{Cay}(G ; S)$.

Subsubsubcase 3.1.2.3. Assume $i=0$ and $k \neq 0$. This means $y z \in S$. We may assume the third element of $S$ does not belong to $\langle x, y, z\rangle$ (otherwise a previous subsubsubcase applies, since $S \cap\langle x, z\rangle=\emptyset$ ). Then the third element of $S$ must be of the form $f x z^{k}$. It is easy to see that

$$
\left((y z)^{2}, f,(y z)^{-2}, f x z^{k}\right) \quad \text { and } \quad\left((y z)^{-2}, f,(y z)^{2}, f x z^{k}\right)
$$

are hamiltonian cycles in $\operatorname{Cay}(G /\langle x, z\rangle ; S)$. Since one or the other of

$$
(y z)^{2} f(y z)^{-2}\left(f x z^{k}\right)=x z^{k+4} \quad \text { and } \quad(y z)^{-2} f(y z)^{2}\left(f x z^{k}\right)=x z^{k-4}
$$

generates $\langle x, z\rangle$, we see from Corollary 2.8 that either

$$
\left((y z)^{2}, f,(y z)^{-2}, f x z^{k}\right)^{3 p} \quad \text { or } \quad\left((y z)^{-2}, f,(y z)^{2}, f x z^{k}\right)^{3 p}
$$

is a hamiltonian cycle in $\operatorname{Cay}(G ; S)$.

Subcase 3.2. Assume $G=D_{6} \times\left(\mathbb{Z}_{3} \ltimes \mathbb{Z}_{p}\right)$. Note that this implies $z^{f}=z$ and $z^{y}=$ $z^{r}$, where $r^{3} \equiv 1(\bmod p)$ and $r \neq 1$. (We must have $p \equiv 1(\bmod 3)$.)

Suppose there exists $s \in S$ whose projection to the second factor is a nontrivial element of $\mathbb{Z}_{p}$. We may assume the first component is a reflection (otherwise it generates a normal subgroup $\left\langle x^{i} z\right\rangle$ which clearly has a trivial intersection with the center of $G$, so Lemma 2.27 applies). That is, $s=f x^{i} z$. Clearly we can assume $s=f z \in S$ (conjugate by a power of $x$ ). Then $s$ yields a double edge in $G / \mathbb{Z}_{p}$, so, by Corollary 2.11 , we may assume $S-\{s\}$ generates $G / \mathbb{Z}_{p}$. From the minimality of $S$, we know $\langle S-\{s\}\rangle \neq G$, so we conclude that $\langle S-\{s\}\rangle=D_{6} \times \mathbb{Z}_{3}$ (or a conjugate). Furthermore, since $|G|$ is the product of only four primes, and $|s|$ is divisible by two of them, we know $|S| \leq 3$. Therefore, some element $t$ of $S-\left\{s_{1}\right\}$ must project nontrivially to both $D_{6}$ and $\langle y\rangle$. Since $\langle s, t\rangle \neq G$, the projection of $t$ to $D_{6}$ must be $f$, so we may assume $t=f y$. Then the final element of $S$ must be of the form $f x^{i}$, with $i \not \equiv 0(\bmod 3)$. Therefore, we may assume $S=\{f z, f y, f x\}$. In this case, Lemma 2.18 applies with $s_{1}=f y$ and $s_{2}=f z$, because $s_{1} s_{2}=y z$ has order 3 , and $\left\langle S-\left\{s_{1}\right\}\right\rangle=\langle f, x, z\rangle$ has order $6 p$.

We may now assume that

the projection of a generator to the second factor is never a nontrivial element of $\mathbb{Z}_{p}$.

Subsubcase 3.2.1. Assume $|S|=2$. The generating set of $\mathbb{Z}_{3} \ltimes \mathbb{Z}_{p}$ must be of the form $\{y, y z\}$, and the generating set of $D_{6}$ is either two reflections or a rotation and a reflection. We now discuss each of the possibilities individually:

Subsubsubcase 3.2.1.1. Let assume that $S=\{f y, f x y z\}$. Since $f y$ generates the cyclic group $G /\langle x z\rangle$, it is obvious that $\left((f y)^{5}, f x y z\right)$ is a hamiltonian cy- 
cle in $\operatorname{Cay}(G /\langle x z\rangle ; S)$. Then, since $(f y)^{5}(f x y z)=x z$, Corollary 2.8 tells us that $\left((f y)^{5}, f x y z\right)^{3 p}$ is a hamiltonian cycle in $\operatorname{Cay}(G ; S)$.

Subsubsubcase 3.2.1.2. Assume $S=\{f y, x y z\}$. Much as in the previous paragraph, it is easy to see that

$$
\left((x y z)^{2},(f y)^{-1},(x y z)^{-2}, f y\right)
$$

is a hamiltonian cycle in $\operatorname{Cay}(G /\langle x z\rangle ; S)$. Therefore, since

$$
(x y z)^{2}(f y)^{-1}(x y z)^{-2}(f y)=x^{4} z^{r-1}
$$

generates $\langle x z\rangle$, Corollary 2.8 tells us that

$$
\left((x y z)^{2},(f y)^{-1},(x y z)^{-2}, f y\right)^{3 p}
$$

is a hamiltonian cycle in $\operatorname{Cay}(G ; S)$.

Subsubcase 3.2.2. Assume $|S|=3$.

Subsubsubcase 3.2.2.1. Assume $S \cap\left(D_{6} \times \mathbb{Z}_{p}\right) \neq \emptyset$. Then (from (9.2) and the fact that $S \cap\langle x, z\rangle=\emptyset$ ) we may assume $f \in S$. There must be an element whose projection to both $D_{6}$ and $\mathbb{Z}_{3} \ltimes \mathbb{Z}_{p}$ is nontrivial. Since by assumption $S$ does not contain any element of the form $f^{\ell} x^{i} z^{k}$ with $k \neq 0$, we are left with two possibilities.

- Assume $f x y \in S$. Because $S$ generates $G$, the third element of $S$ must be of the form $f^{\ell} x^{i} y z$ (or its inverse). Since $S$ is minimal, this element must either be $y z$ or $f x y z$. Thus, $S$ is either $\{f, f x y, y z\}$ or $\{f, f x y, f x y z\}$. In either case, taking $s_{1}=f$ and $s_{2}=f x y$ we get that $s_{1} s_{2}=x y$ is of order 3 and $\left\langle S-\left\{s_{1}\right\}\right\rangle=\langle f x, y, z\rangle$ is of order $6 p$, so clearly Lemma 2.18 applies.

- Assume $x y \in S$. Since $S$ generates $G$, the third element of $S$ must be of the form $f^{\ell} x^{i} y z$ (or its inverse). Since $S$ is minimal, we must have $\ell=0$. There are three Cayley graphs to consider:

○ Suppose $S=\{f, x y, y z\}$. Taking $s_{1}=(x y)^{-1}=x^{2} y^{2}$ and $s_{2}=y z$, we see that $s_{1} s_{2}=x^{2} z$ is of order $3 p$ and clearly $\left|\left\langle S-\left\{s_{1}^{-1}\right\}\right\rangle\right|=6$. So Lemma 2.18 applies.

○ Suppose $S=\{f, x y, x y z\}$. Taking $s_{1}=(x y)^{-1}=x^{2} y^{2}$ and $s_{2}=x y z$, we see that $s_{1} s_{2}=z$ is of order $p$, and $\left\langle S-\left\{s_{1}^{-1}\right\}\right\rangle=\langle f, x, y z\rangle$ has order 18. So Lemma 2.18 applies.

○ Suppose $S=\left\{f, x y, x^{2} y z\right\}$. Since $G /\langle x, z\rangle$ is abelian, it is easy to see that

$$
\left(f,(x y)^{-2}, f, x y, x^{2} y z\right)
$$

is a hamiltonian cycle in $\operatorname{Cay}(G /\langle x, z\rangle ; S)$. Therefore, since

$$
(f)(x y)^{-2}(f)(x y)\left(x^{2} y z\right)=x^{2} z
$$

generates $\langle x z\rangle$, Corollary 2.8 tells us that

$$
\left(f,(x y)^{-2}, f, x y, x^{2} y z\right)^{3 p}
$$

is a hamiltonian cycle in $\operatorname{Cay}(G ; S)$. 
Subsubsubcase 3.2.2.2. Assume $S \cap\left(D_{6} \times \mathbb{Z}_{p}\right)=\emptyset$. We must have an element of the form $f x^{i} y z^{k}$ in $S$, so we can assume

$$
f y \in S
$$

In order to generate $D_{6}$, the set $S$ must also contain an element of the form $f x y^{j} z^{k}$ or $x y^{j} z^{k}$. Furthermore, the assumption of this paragraph implies $j \neq 0$, so we may assume $j=1$, by passing to the inverse if necessary.

- Suppose $f x y z^{k} \in S$. Since $S$ is minimal and $|S|=3$, we must have $k=0$; that is, $f x y \in S$. Then, because $S$ generates $G$, the third element of $S$ must be of the form $f^{\ell} x^{i} y z$. If $i \neq 0$, then $\left\langle f y, f^{\ell} x^{i} y z\right\rangle=G$, while if $i=0$ and $\ell=1$, then $\langle f x y, f y z\rangle=G$. These conclusions contradict the minimality of $S$, so there is only one Cayley graph to consider: we have $S=\{f y, f x y, y z\}$. Taking $s_{1}=f x y$ and $s_{2}=(f y)^{-1}=f y^{2}$, we get that $s_{1} s_{2}=x^{2}$ is of order 3 , and $\left\langle S-\left\{s_{1}\right\}\right\rangle=\langle f, y, z\rangle$ is clearly of order $6 p$. So Lemma 2.18 applies.

- Suppose $x y z^{k} \in S$. Since $S$ is minimal and $|S|=3$ we must have $k=0$, so $x y \in S$. Then, because $S$ generates $G$, the third element of $S$ must be of the form $f^{\ell} x^{i} y z$. If $i \neq 0$, then $\left\langle f y, f^{\ell} x^{i} y z\right\rangle=G$, while if $i=0$ and $\ell=1$, then $\langle x y, f y z\rangle=G$. So there is only one Cayley graph to consider: we have $S=\{f y, x y, y z\}$. Taking $s_{1}=f y$ and $s_{2}=(x y)^{-1}=x^{2} y^{2}$, we get that $s_{1} s_{2}=f x^{2}$ is of order 2 and $\left\langle S-\left\{s_{1}\right\}\right\rangle=\langle x, y, z\rangle$ is of order $9 p$. So Lemma 2.18 applies.

Subsubcase 3.2.3. Assume $|S|=4$. Since $|G|=18 p$ is the product of only four prime factors, the order of the subgroup generated by any two elements of $S$ must be the product of only two prime factors. It is easy to see that this implies every element of $S$ belongs to either $D_{6} \times\{e\}$ or $\{e\} \times\left(\mathbb{Z}_{3} \ltimes \mathbb{Z}_{p}\right)$. Therefore, Cay $(G ; S)$ is isomorphic to

$$
\operatorname{Cay}\left(D_{6} ; S_{1}\right) \times \operatorname{Cay}\left(\mathbb{Z}_{3} \ltimes \mathbb{Z}_{p} ; S_{2}\right) .
$$

Since the Cartesian product of hamiltonian graphs is hamiltonian, we may conclude that $\operatorname{Cay}(G ; S)$ has a hamiltonian cycle.

Subcase 3.3. Assume $G=\left(D_{6} \times \mathbb{Z}_{3}\right) \ltimes \mathbb{Z}_{p}$, where $D_{6}$ and $\mathbb{Z}_{3}$ both act nontrivially on $\mathbb{Z}_{p}$. (Note that we must have $p \equiv 1(\bmod 3)$.) This implies $z^{f}=z^{-1}$ and $z^{y}=z^{r}$ where $r^{3} \equiv 1(\bmod p)($ but $r \not \equiv 1(\bmod p))$.

Subsubcase 3.3.1. Assume $|S|=2$.

Subsubsubcase 3.3.1.1. Assume $S \cap\langle f, x, z\rangle=\emptyset$. The generating set $S$ must contain an element of the form $f x^{i} y^{j} z^{k}$. By assumption, we must have $j \neq 0$, so we may assume $j=1$. Then, conjugating by an element of $\langle x, z\rangle$, we may assume $f y \in S$.

To generate $G$, the second element of $S$ must be of the form $f^{\ell} x y^{j^{\prime}} z$. By assumption, we must have $j^{\prime} \neq 0$, so we may assume $j^{\prime}=1$. Therefore, there are only two possibilities, and we discuss each of them individually:

- Suppose $S=\{f y, x y z\}$. Since $\left((f y)^{-1},(x y z)^{-2}, f y,(x y z)^{2}\right)$ is a hamiltonian cycle in $\operatorname{Cay}(G /\langle x, z\rangle ; S)$, and

$$
(f y)^{-1}(x y z)^{-2}(f y)(x y z)^{2}=x z^{(r+1)^{2}} \text { generates }\langle x, z\rangle,
$$


Corollary 2.8 tells us $\left((f y)^{-1},(x y z)^{-2}, f y,(x y z)^{2}\right)^{3 p}$ is a hamiltonian cycle in $\operatorname{Cay}(G ; S)$.

- Suppose $S=\{f y, f x y z\}$. Since $f x y z \equiv f y(\bmod \langle x, z\rangle)$, it is obvious that $\left((f y)^{5}, f x y z\right)$ is a hamiltonian cycle in $\operatorname{Cay}(G /\langle x, z\rangle ; S)$. Then, since the element $(f y)^{5}(f x y z)=x z$ generates $\langle x, z\rangle$, Corollary 2.8 tells us that $\left((f y)^{5}, f x y z\right)^{3 p}$ is a hamiltonian cycle in $\operatorname{Cay}(G ; S)$.

Subsubsubcase 3.3.1.2. Assume $S \cap\langle f, x, z\rangle \neq \emptyset$. Since $S \cap\langle x, z\rangle=\emptyset$, we must have $S \cap f\langle x, z\rangle \neq \emptyset$. Then, conjugating by an element of $\langle x, z\rangle$, we may assume $f \in S$. To generate $G$, the second element of $S$ must be of the form $f^{\ell} x y z$.

- Suppose $S=\{f, x y z\}$. Since $\left(f,(x y z)^{-2}, f,(x y z)^{2}\right)$ is a hamiltonian cycle in $\operatorname{Cay}(G /\langle x, z\rangle ; S)$, and

$$
f(x y z)^{-2} f(x y z)^{2}=x z^{2(r+1)} \text { generates }\langle x, z\rangle,
$$

Corollary 2.8 tells us that $\left(f,(x y z)^{-2}, f,(x y z)^{2}\right)^{3 p}$ is a hamiltonian cycle in the graph $\operatorname{Cay}(G ; S)$.

- Suppose $S=\{f, f x y z\}$. We may assume $4 r \not \equiv-5(\bmod p)$ (by replacing $y$ with its inverse, if necessary). Let

$$
\begin{aligned}
& \left(s_{i}\right)_{i=1}^{18}= \\
& \quad\left(f x y z, f,(f x y z)^{-2}, f,(f x y z)^{-3}, f,(f x y z)^{3}, f,(f x y z)^{2}, f,(f x y z)^{-1}, f\right) .
\end{aligned}
$$

Using the fact that $r^{2}+r+1 \equiv 0(\bmod p)$, we calculate that the vertices of this walk are:

$$
\begin{aligned}
& e, f x y z, x^{2} y z^{-1}, f x^{2} z^{-2 r-2}, x^{2} y^{2} z^{-3 r-1}, f x y^{2} z^{3 r+1}, y z^{-3} \\
& \quad f x z^{-4 r-4}, y^{2} z^{-5 r-1}, f y^{2} z^{5 r+1}, x z^{4 r+6}, f y z^{5-2 r}, x y^{2} z^{-7 r-1} \\
& f x^{2} y^{2} z^{7 r+1}, x^{2} z^{6 r+8}, f x^{2} y z^{7-2 r}, x y z^{2 r-7}, f z^{-8 r-10}, z^{8 r+10}
\end{aligned}
$$

Then, by modding out $\langle z\rangle$, we see that this walk visits the vertices of $G / \mathbb{Z}_{p} \cong$ $D_{6} \times \mathbb{Z}_{3}$ in the order

$$
e, f x y, x^{2} y, f x^{2}, x^{2} y^{2}, f x y^{2}, y, f x, y^{2}, f y^{2}, x, f y, x y^{2}, f x^{2} y^{2}, x^{2}, f x^{2} y, x y, f, e,
$$

so it is a hamiltonian cycle in $\operatorname{Cay}\left(G / \mathbb{Z}_{p} ; S\right)$. Furthermore, from our assumption that $4 r \not \equiv-5(\bmod p)$, we see that the final vertex $z^{8 r+10}$ is not trivial in $G$, so it generates $\langle z\rangle$. Therefore Corollary 2.8 provides a hamiltonian cycle in $\operatorname{Cay}(G ; S)$.

Subsubcase 3.3.2. Assume $|S|=3$.

Subsubsubcase 3.3.2.1. Assume some element of $S$ has order 6 . Then $S$ contains $f y$ (or a conjugate). The only proper subgroups of $G$ that properly contain $f y$ are $\langle f\rangle \times\left(\mathbb{Z}_{3} \ltimes \mathbb{Z}_{p}\right)$ and $D_{6} \times\langle y\rangle$. Thus, recalling the assumption that $S \cap\langle x, z\rangle=\emptyset$ :

- the second generator can be assumed to be $y z, f z$, or $f y z$, and 
- the third generator can be assumed to be $f x, f x y$, or $x y$.

We consider each possible choice of the second generator.

a. If $y z \in S$, then any of the possible third generators can be used:

- Suppose $S=\{f y, y z, f x\}$. Taking $s_{1}=f y$ and $s_{2}=f x$ we get that $s_{1} s_{2}=$ $x y$ is of order 3 and since $\left\langle S-\left\{s_{1}\right\}\right\rangle=\langle f x, y, z\rangle$ has order $6 p$, it is easy to see that Lemma 2.18 applies.

- Suppose $S=\{f y, y z, f x y\}$. Taking $s_{1}=f y$ and $s_{2}=f x y$ we get that $s_{1} s_{2}=x y^{2}$ is of order 3 and since $\left\langle S-\left\{s_{1}\right\}\right\rangle=\langle f x, y, z\rangle$ has order $6 p$, it is easy to see that Lemma 2.18 applies.

- Suppose $S=\{f y, y z, x y\}$. Taking $s_{1}=f y$ and $s_{2}=(x y)^{-1}$ we get that $s_{1} s_{2}=f x^{2}$ is of order 2 and since $\left\langle S-\left\{s_{1}\right\}\right\rangle=\langle x, y, z\rangle$ has order $9 p$, it is easy to see that Lemma 2.18 applies.

b. If $f z \in S$, then, because $\langle f z, f x y\rangle=G=\langle f z, x y\rangle$, there is only one possibility for the third generator: we have $S=\{f y, f z, f x\}$. Taking $s_{1}=f x$ and $s_{2}=f y$ we get that $s_{1} s_{2}=x^{2} y$ is of order 3 and since $\left\langle S-\left\{s_{1}\right\}\right\rangle=\langle f, y, z\rangle$ has order $6 p$, it is easy to see that Lemma 2.18 applies.

c. If $f y z \in S$, then, because $\langle f y z, f x\rangle,\langle f y z, f x y\rangle$, and $\langle f y z, x y\rangle$ are all equal to $G$, none of the possible third generators yield a minimal generating set of $G$. So there are no Cayley graphs to consider in this case.

Subsubsubcase 3.3.2.2. Assume no element of $S$ has order 6 . Then $S$ contains $f$ (or a conjugate). There must be an element of $S$ that does not belong to $D_{6} \times \mathbb{Z}_{p}$ (that is, an element of the form $\left.f^{\ell} x^{i} y z^{k}\right)$. Because there is no element of order 6 , we must have $\ell=0$, so the possibilities are: $y, y z, x y$, and $x y z$. However, we eliminate the last option, because $\langle f, x y z\rangle=G$.

We consider each of the remaining possibilities:

a. Suppose $y \in S$. The third generator must involve both $x$ and $z$. Since $\langle f, x y z\rangle=G$ and since we assumed $S \cap\langle x, z\rangle=\emptyset$, there is only one possibility, namely, $S=$ $\{f, y, f x z\}$. Taking $s_{1}=f x z$ and $s_{2}=f$ we get that $s_{1} s_{2}=x^{2} z^{-1}$ is of order $3 p$ and since $\left\langle S-\left\{s_{1}\right\}\right\rangle=\langle f, y\rangle$ has order 6 , it is easy to see that Lemma 2.18 applies.

b. Suppose $y z \in S$. The third generator must involve $x$. Since $S$ does not contain an element of order 6 , or any element of $\langle x, z\rangle$, and $\left\langle f, x y z^{k}\right\rangle=G$ for $k \neq 0$, the only possibilities are $f x z^{k}$ and $x y$.

- Suppose $S=\left\{f, y z, f x z^{k}\right\}$. Note that, because

$$
2\left(r^{2}+r\right)+2(r+1)=2(r+1)^{2} \not \equiv 0(\bmod p),
$$

it cannot be the case that $k+2\left(r^{2}+r\right)$ and $k-2(r+1)$ are both 0 modulo $p$. Therefore, $\langle x, z\rangle$ is generated by either

$$
\begin{aligned}
& (y z)^{2}(f)(y z)^{-2}\left(f x z^{k}\right)=x z^{k+2\left(r^{2}+r\right)} \quad \text { or } \\
& (y z)^{-2}(f)(y z)^{2}\left(f x z^{k}\right)=x z^{k-2(r+1)},
\end{aligned}
$$


so Corollary 2.8 tells us that either

$$
\left((y z)^{2}, f,(y z)^{-2}, f x z^{k}\right)^{3 p} \text { or }\left((y z)^{-2}, f,(y z)^{2}, f x z^{k}\right)^{3 p}
$$

is a hamiltonian cycle in $\operatorname{Cay}(G ; S)$.

- Suppose $S=\{f, y z, x y\}$. Since $(x y)^{-2} f(y z)^{2} f=x z^{-(r+1)}$ generates $\langle x, z\rangle$, Corollary 2.8 tells us that

$$
\left((x y)^{-2}, f,(y z)^{2}, f\right)^{3 p}
$$

is a hamiltonian cycle in $\operatorname{Cay}(G ; S)$.

c. Suppose $x y \in S$. The third generator must involve $z$. However, $\left\langle x y, f x^{i} y^{j} z\right\rangle=G$, and $\left\langle f, x^{i} y z\right\rangle$ is also equal to $G$ if $i \neq 0$. Since there is no element of the form $x^{i} z^{k}$ in $S$, this implies that the only possibility for the third generator is $y z$, so $S=\{f, x y, y z\}$, but this generating set was already considered in the preceding paragraph.

Subsubcase 3.3.3. Assume $|S|=4$. Some 3-element subset $S^{\prime}$ of $S$ must generate $G / \mathbb{Z}_{p}$. Then, because $S$ is minimal, we must have $\left\langle S^{\prime}\right\rangle=D_{6} \times \mathbb{Z}_{3}$ (or a conjugate). Since $S^{\prime}$ must be minimal, and $S^{\prime} \cap\langle x\rangle=\emptyset$, we must have $S^{\prime}=\{f, f x, y\}$.

Now the final element of $S$ must be of the form $f^{\ell} x^{i} y^{j} z$. Since $S \cap\langle x, z\rangle=\emptyset$, we know that $\ell$ and $j$ cannot both be 0 .

- If $\ell \neq 0$, then either $\left\langle f x, y, f^{\ell} x^{i} y^{j} z\right\rangle=G$, or $\left\langle f, y, f^{\ell} x^{i} y^{j} z\right\rangle=G$, depending on whether $i$ is 0 or not.

- If $j \neq 0$, then $\left\langle f, f x, f^{\ell} x^{i} y^{j} z\right\rangle=G$.

These conclusions contradict the minimality of $S$, so there are no Cayley graphs to consider in this case.

Acknowledgments. We are grateful to D. Jungreis, E. Friedman, and J. A. Gallian for making the unpublished manuscript [14] available to us. We also thank two anonymous referees for their helpful comments.

D. W. M. was partially supported by a research grant from the Natural Sciences and Engineering Research Council of Canada. K. K., D. M., and P. Š. were partially supported by Agencija za raziskovalno dejavnost Republike Slovenije, research program P1-0285.

\section{References}

[1] B. Alspach, The classification of hamiltonian generalized Petersen graphs, J. Combin. Theory B 34 (1983), 293-312.

[2] B. Alspach, Lifting Hamilton cycles of quotient graphs, Discrete Math. 78 (1989), 25-36.

[3] B. Alspach, C. C. Chen and M. Dean, Hamilton paths in Cayley graphs on generalized dihedral groups, Ars Math. Contemp. 3 (2010), 29-47.

[4] C. C. Chen and N. Quimpo, On strongly hamiltonian abelian group graphs, in: K. L. McAvaney (ed.), Combinatorial Mathematics VIII (Proceedings, Geelong, Australia 1980), SpringerVerlag, Berlin, 1981, pp. 23-24. 
[5] C. C. Chen and N. Quimpo, Hamiltonian Cayley graphs of order $p q$, in: Combinatorial mathematics, X (Adelaide, 1982), Springer, Berlin, 1983, pp. 1-5.

[6] S. J. Curran and J. A. Gallian, Hamiltonian cycles and paths in Cayley graphs and digraphs-a survey, Discrete Math. 156 (1996), 1-18.

[7] S. J. Curran, J. Morris and D. W. Morris: Cayley graphs of order $16 p$ are hamiltonian (preprint), http://arxiv.org/abs/1104.0081.

[8] J. A. Gallian, Contemporary Abstract Algebra, 6th edition, Houghton Mifflin, Boston, 2006.

[9] E. Ghaderpour and D. W. Morris, Cayley graphs of order $27 p$ are hamiltonian (preprint), http://arxiv.org/abs/1101.4322.

[10] E. Ghaderpour and D. W. Morris, Cayley graphs of order $30 p$ are hamiltonian (preprint), http://arxiv.org/abs/1102.5156.

[11] D. Gorenstein, Finite Groups, Chelsea, New York, 1980.

[12] J. L. Gross and T. W. Tucker, Topological Graph Theory, Wiley, New York, 1987.

[13] M. Hall, The Theory of Groups, Macmillan, New York, 1959.

[14] D. Jungreis and E. Friedman, Cayley graphs on groups of low order are hamiltonian (unpublished).

[15] K. Keating and D. Witte, On Hamilton cycles in Cayley graphs with cyclic commutator subgroup, Ann. Discrete Math. 27 (1985), 89-102.

[16] K. Kutnar and D. Marušič, Hamilton paths and cycles in vertex-transitive graphs - current directions, Discrete Math. 309 (2009), 5491-5500.

[17] K. Kutnar, D. Marušič, J. Morris, D. W. Morris and P. Šparl: Cayley graphs on $A_{5}$ are hamiltonian (unpublished), http://arxiv.org/abs/1009.5795/anc/A5.pdf.

[18] K. Kutnar and P. Šparl, Hamilton paths and cycles in vertex-transitive graphs of order $6 p$, Discrete Math. 309 (2009), 5444-5460.

[19] D. Li, Cayley graphs of order pqr are Hamiltonian (Chinese), Acta Math. Sinica 44 (2001), 351-358.

[20] S. C. Locke and D. Witte, Flows in circulant graphs of odd order are sums of Hamilton cycles, Discrete Math. 78 (1989), 105-114.

[21] D. W. Morris, 2-generated Cayley digraphs on nilpotent groups have hamiltonian paths (preprint), http://arxiv.org/abs/1103.5293.

[22] I. Pak and R. Radoičić, Hamiltonian paths in Cayley graphs, Discrete Math. 309 (2009), 55015508.

[23] C. Savage, A survey of combinatorial Gray codes, SIAM Review 39 (1997), 605-629.

[24] H. Wielandt, Finite Permutation Groups, Academic Press, New York,1964.

[25] D. Witte, On Hamiltonian circuits in Cayley diagrams, Discrete Math. 38 (1982), 99-108.

[26] D. Witte, Cayley digraphs of prime-power order are hamiltonian, J. Comb. Th. B $\mathbf{4 0}$ (1986), 107-112.

[27] D. Witte and J. A. Gallian, A survey: Hamiltonian cycles in Cayley graphs, Discrete Math. 51 (1984), 293-304. 